\title{
Maximally hyperbolic operators
}

\author{
By
}

\section{TAKASHI ŌKAJI}

\section{Introduction and statement of results}

Let $\Omega$ be a domain in $\mathbf{R}^{n}$ and $I$ be an interval in $\mathbf{R}$. For $(x, t) \in \Omega \times I$, we consider the following differential operator with smooth coefficients:

$$
P\left(x, t, D_{x}, D_{t}\right)=D_{t}^{m}+\sum_{|\alpha|+j \leq m, j \leq m-1} a_{\alpha, j}(x, t) D_{x}^{\alpha} D_{t}^{j},
$$

where $D_{x}=\sqrt{-1}^{-1} \partial_{x}$, etc.. We are concerned with the well-posedness for the Cauchy problem for $P$ in the $C^{\infty}$ class with the initial surface $\left\{t=t_{0}\right\}$.

For real $s$, we denote the standard Sobolev spaces over $\mathbf{R}^{n+1}$ by $H^{s}$ and its norm by $\|\cdot\|_{s}$ respectively. We say that the operator $P$ is strictly hyperbolic if and only if its characteristic roots are real and distinct. It is a classical fact that if $P$ is strictly hyperbolic, then there are some real number $\gamma_{0}$ and a positive constant $C$ such that

$$
r\left\|e^{-r t} u\right\|_{m-1} \leq C\left\|e^{-r t} P u\right\|_{0}
$$

for any $\gamma>\gamma_{0}$ and $u \in C_{0}^{\infty}(K \times I)$, where $K$ is a compact subset of $\Omega$.

The purpose of this paper is to give a generalization of this fact with respect to the regularity property of the solution. In fact, we shall introduce a notion of maximal hyperbolicity and give a sufficient condition for $P$ to be maximally hyperbolic.

To make the statement more precisely, we must recall some class of operators. Let $\Sigma$ be a conic submanifold of the cotangent space $T^{*}(\Omega) \backslash\{0\}$ with codimension $p$. For a real $m$ and a non-negative integer $k$, we denote by $\mathcal{N}^{m, k}$ $(\Omega, \Sigma)$ a space of pseudo-differential operators op $(p)$, introduced in [B], [ $\mathrm{BGH}]$, such that $p(x, \xi) \in S_{1,0}^{\mathrm{m}}$ and

$$
p(x, \xi) \sim \sum_{j=0}^{\infty} p_{m-j}(x, \xi),
$$

where $p_{m-j}$ is positively homogeneous of degree $m-j$ in $\xi$ and vanishes with order $k-2 j$ on $\Sigma$ if $k-2 j \geq 0$ : i.e. for some positive constant $C$ and for $k-2 j \geq$ 0 ,

$$
\left|p_{m-j}(x, \xi)\right| \leq C|\xi|^{m-k+j} d_{\Sigma}(x, \xi)^{k-2 j}
$$


where $d_{\Sigma}(x, \xi)$ is a positively homogeneous function of degree 1 which is equal to the distance between $(x, \xi)$ and $\Sigma$ when $|\xi|=1$. When $m=k$, we simply write it by $\mathcal{N}^{\mathrm{m}}$. For $P \in \mathcal{N}^{m, k}(\Omega, \Sigma)$, we define the localization of $P$ by

$$
\sigma_{x, \xi}^{k}(P)=\sum_{|\alpha+\beta|+2 j=k} \frac{1}{\alpha ! \beta !} \partial_{x}^{\alpha} \partial_{\xi}^{\beta} p_{j}(x, \xi) y^{\alpha} D_{y}^{\beta} .
$$

We consider the following operators

$$
P=D_{t}^{m}+\sum_{j=1}^{m} A_{j}(x, D) D_{t}^{m-j},
$$

where $A_{j} \in \mathcal{N}^{j}(\Omega, \Sigma)$.

Now, we say that an operator given by (1.1) is maximally hyperbolic in $\Omega \times I$ if and only if for any compact subset $K$ of $\Omega$ and $B_{j} \in \mathcal{N}^{j}$, there are a real number $\gamma_{0}$ and a positive constant $C$ such that

$$
\gamma \sum_{j=0}^{m-1}\left\|e^{-\gamma t} B_{j} D_{t}^{m-1-j} u\right\|_{0} \leq C \|_{e^{-\gamma t} P u \|_{0}},
$$

when $\gamma>\gamma_{0}$ and $u \in C_{0}^{\infty}(K \times I)$.

We make the following assumptions:

(A-1) The restriction to $\Sigma$ of the canonical 2 -form $\sum_{j=1}^{n} d \xi_{j} \wedge d x_{j}$ on $T^{*}(\Omega)$ has a constant rank $p$ at every point of $\Sigma$ and $A_{m} \in \mathcal{N}^{m}(\Omega, \Sigma)$ is a transversally elliptic: for some positive constant $C$,

$$
d_{\Sigma}(x, \xi)^{m} \leq C\left|a_{m}(x, \xi)\right| .
$$

(A-2) Denoting the principal symbol of $A_{j}$ by $a_{j}(x, \xi)$, we assume that the polynomial in $\tau$

$$
p(\tau, x, \xi) \tau^{m}+\sum_{j=0}^{m-1} a_{m-j}(x, \xi) \tau^{j}
$$

has only real roots which are pairwise distinct if $(x, \xi) \notin \Sigma$.

$(\mathrm{A}-3) \quad$ For the operator

$$
\begin{aligned}
& \mathscr{P}_{x, \xi}(\tau)=\tau^{m}+\sum_{j=0}^{m-1} \sigma_{x, \xi}^{m-j}\left(A_{m-j}\right) \tau^{j}, \\
& \partial_{\zeta} \mathscr{P}_{x, \xi}^{*}(\zeta) \mathscr{P}_{x, \xi}(\zeta)-\mathscr{P}_{x, \xi}^{*}(\zeta) \partial_{\zeta} \mathscr{P}_{x, \xi}(\zeta)=0,
\end{aligned}
$$

if $\zeta \in \mathbf{R}$ and $(x, \xi) \in \Sigma$.

$(\mathrm{A}-4)$ For any $\zeta \in \mathbf{C}$ with $\operatorname{Im} \zeta<0$ and any $(x, \xi) \in \Sigma, \mathscr{P}_{x, \xi}(\zeta)$ is injective in $\mathscr{S}\left(\mathbf{R}^{n}\right)$ : i.e. there is a positive constant $C$ such that

$$
\|f\| \leq C\left\|\Phi_{x, \xi}(\zeta) f\right\|
$$

for any $f \in \mathscr{S}\left(\mathbf{R}^{n}\right)$ and moreover, for each $\sigma \in \mathbf{R}$ and $(x, \xi) \in \Sigma$, there is a positive constant $\delta$ such that for any $f \in \mathscr{S}\left(\mathbf{R}^{n}\right)$,

$$
\liminf _{\gamma \rightarrow 0-}\left\|\gamma^{-1} \mathscr{P}_{x, \xi}(\sigma+i \gamma) f\right\| \geq \delta\|f\| .
$$


Here, $\|\cdot\|$ means $L^{2}\left(\mathbf{R}^{n}\right)$-norm.

Theorem 1.1. For the operator $P$ given by (1.1), we assume (A-1) (A-4). Then, $P$ is maximally hyperbolic in $\Omega \times I$.

The proof will be given in the following sections. It is based on the same principle as in $[\mathrm{O}]_{3}$ but requires some technical complications. Our methods are closed to those of Chap. $\mathrm{X}$ in $[\mathrm{HN}]_{2}$ and $[\mathrm{BGH}]$ in which they have investigated the maximal hypoellipticity. In $\S 6$, we shall give an example which satisfies our conditions. In $\S 7$, we shall discuss the necessary condition for $P$ to be maximally hyperbolic.

\section{Notation and classes of symbols}

We denote the class of standard pseudo-differential operators of $(\rho, \delta)$ type by

$O P S_{\rho, \delta}^{m}(\Omega)$, or simply, by $O P S_{\rho, \delta}^{m}$.

For $a \in S_{\rho, \delta}^{m}$, we write

$$
a_{(\beta)}^{(\alpha)}(x, \xi)=\partial_{\xi}^{\alpha} \partial_{x}^{\beta} a(x, \xi)
$$

and denote by op $(a)$ the corresponding pseudo-differential operator

$$
\text { op }(a) u(x)=(2 \pi)^{-n} \int e^{-i x \cdot \xi} a(x, \xi) \hat{u}(\xi) d \xi \text {. }
$$

Following [BGH], we introduce a system of coordinates $(u, v)$ in $T^{*}(\Omega)$ and classes of symbols, attached to $\Sigma$. Let $(x, \xi)$ be a point of $\Sigma$. Then there are a conic neighborhood $\Theta$ and $C^{\infty}$-functions $u_{i}, \quad 1 \leq i \leq p$, positively homogeneous of degree 0 such that $\left\{d u_{j}\right\}$ are linearly independent and

$$
\Sigma \cap \Theta=\left\{u_{1}=u_{2}=\cdots=u_{p}=0\right\} \text {. }
$$

Also, we can find positively homogeneous $C^{\infty}$-functions $v_{i}, 1 \leq i \leq 2 n-p$, with degree 1 such that $(u, v)$ is a system of coordinate in $\Theta$ (if possibly shrinking it). By $S^{m, k}(\Omega, \Sigma)$ (or simply by $S^{m, k}$ ), we mean the class of symbols chracterized by the following: $a \in S^{m, k}$ is if and only if $a$ is in $S_{1,0}^{m}(\Omega)$ outside of $\Sigma$ and every point of $\Sigma$ has a conic neighborhood in which for any $\alpha, \beta$ and any $|v| \geq 1$, there is a constant $C_{\alpha, \beta}$ such that

$$
\left|\partial_{u}^{\alpha} \partial_{v}^{\beta} a\right| \leq C_{\alpha, \beta}|v|^{m-|\beta|}\left(|u|+|v|^{-1 / 2}\right)^{k-|\alpha|} .
$$

We set $\mathscr{H}^{\mathrm{m}}=\bigcap_{j=0}^{\infty} S^{m-j, k-2 j}$ and denote the classes of pseudo-differential operators corresponding to these classes by $O P S^{m, k}$ and $O P H^{m}$.

For $E=\mathbf{R}^{n}$, we denote by $S^{m}(E)$ the space of $C^{\infty}$-functions $a$ such that for 
any $\alpha$,

$$
(1+|\Xi|)^{-m+|\alpha|} D_{\Xi}^{\alpha} a(\Xi)
$$

is bounded and by $S_{\text {reg }}^{m}(E)$ we mean the space of $C^{\infty}$-functions $a$ such that $a \in$ $S^{m}(E)$ and

$$
a \sim \sum_{k=0}^{\infty} a_{k},
$$

where $a_{k} \in C^{\infty}(E \backslash\{0\})$ is positively homogeneous of degree $m-k$. Here, the asymptotic expansion means that for any non-negative integer $N$, there is a symbol $b_{N} \in S^{m-N}(E)$ such that

$$
a-\sum_{j=0}^{N-1}=b_{N}
$$

if $|\Xi| \geq 1$.

We recall some symbol calculus in $[\mathrm{BGH}]$. Let $N=\mathbf{R}^{p}$ and let $L$ be a linear transformation from $E \times E^{*}$ to $N$. We can write

$$
L(x, \xi)=B x+C \xi
$$

if $x \in E$ and $\xi \in E^{*}$. Let

$$
A=C\left({ }^{t} B\right) \in \mathscr{L}\left(N^{*}, N\right) \text {. }
$$

For a symbol $a \in S^{m}(E)$, we define the corresponding pseudo-differential operator $a_{L}$ by

$$
a_{L} f=(2 \pi)^{-n} \int e^{i x \cdot \xi} a(L(x, \xi)) \hat{f}(\xi) d \xi .
$$

Also, if $E=N, B=I d$ and $C=A$, we simply write $a_{L}=a_{A}$. Generally, for two symbols $a \in S^{m}(E)$ and $b \in S^{m^{\prime}}(E)$,

$$
a_{L} b_{L}=\left(a_{A} b\right)_{L} \text { and } a_{A} b_{A}=\left(a_{A} b\right)_{A} \text {. }
$$

Namely, denoting $c_{L}=a_{L} b_{L}$, we have

$$
c(y)=(2 \pi)^{-n} \int e^{i y \cdot \eta} a(y+A \eta) \hat{b}(\eta) d \eta
$$

and

$$
c(\Xi) \sim \sum_{a \geq 0} \frac{1}{\alpha !} \partial_{\Xi}^{\alpha} a(\Xi)\left(A D_{\Xi}\right)^{\alpha} b(\Xi)
$$

Let $N=\mathbf{R}_{u}^{p}$. Locally we identify $\Sigma$ with $\mathbf{R}_{v}^{2 n-p}$. For a domain $G$ of $\mathbf{R}^{q}$, we say that $a \in C^{\infty}\left(G, S^{m}(N)\right)$ belongs to the class $S^{m}(G \times N)$ if and only if for any compact subset $K$ in $G$ and any multi-index $\alpha, \beta$, there is a positive constant $C_{K, \alpha, \beta}$ such that

$$
\left|\partial_{w}^{\alpha} \partial_{u}^{\beta} \mathrm{a}(w, u)\right| \leq \mathrm{C}_{K, \alpha, \beta}\langle\mathrm{u}\rangle^{m-|\beta|}
$$


for $w \in K$. Here, $\langle u\rangle=\left(1+|u|^{2}\right)^{\frac{1}{2}}$. Also, we assume that

$$
L \in C^{\infty}\left(G, \mathscr{L}\left(E \times E^{*}, N\right)\right) .
$$

Then, for $a \in S^{m}(G, N), a_{L}$ denotes the family of pseudo-differential operators defined by $(2.1)$.

If $P$ is in $\mathcal{N}^{m, k}$, then in a neighborhood $\Theta$ of each point $(x, \xi)$ of $\Sigma$, there are a unique polynomial $T_{k}(P)$

$$
T_{k}(P)=\sum_{|\alpha| \leq k} \mathrm{a}_{\alpha}(v) u^{\alpha}
$$

and two unique differential operators $P_{\Sigma}$ and $P_{\Sigma}^{\prime}$

$$
\begin{aligned}
& P_{\Sigma}=\sum a_{\alpha \beta}(v) u^{\alpha} D_{u}^{\beta} \\
& P_{\Sigma}^{\prime}=\sum b_{\alpha \beta}(v) u^{\alpha} D_{u}^{\beta}
\end{aligned}
$$

such that $P-T_{k}(P)$ vanishes with order $k+1$ on $\Sigma$ and for any $Q \in \mathcal{N}^{m^{\prime}, k^{\prime}}$,

$$
T_{k+k^{\prime}}(P \circ Q)=P_{\Sigma} T_{k^{\prime}}(Q) \text { and } T_{k+k^{\prime}}(Q \circ P)=P_{\Sigma}^{\prime} T_{k^{\prime}}(Q)
$$

where $a_{\alpha}(v)$ and $a_{\alpha \beta}(v)\left(b_{\alpha \beta}(v)\right)$ are smooth functions depending only on $v$ and are positively homogeneous of degree $m-\frac{1}{2}(k-|\alpha|)$ and $m-\frac{1}{2}(k-|\alpha|+$ $|\beta|)$, respectively.

Among these localized objects, there are some relations. Let $y \in \mathbf{R}^{n}$ and $\eta$ be its dual variable. We define two $p \times n$-matrices $B$ and $C$ by

$$
B=\left(B_{j k}\right)_{1 \leq j \leq p, 1 \leq k \leq n,}, \mathrm{~B}_{j k}(v)=\left.\frac{\partial u_{j}}{\partial x_{k}}\right|_{\Sigma}
$$

and

$$
C=\left(C_{j k}\right)_{1 \leq j \leq p, 1 \leq k \leq n,}, C_{j k}(v)=\left.\frac{\partial u_{j}}{\partial \xi_{k}}\right|_{\Sigma},
$$

respectively. Let

$$
L(y, \eta)=B y+C \eta \text { and } A=C\left({ }^{t} B\right) .
$$

Then, $L \in C^{\infty}(\Sigma, N)$ and $a=T_{k}(P) \in S^{k}(\Sigma, N)$. Proposition 5.14 in [BGH] says that for this symbol $a$,

$$
P_{\Sigma}=a_{A}, P_{\Sigma}^{\prime}=a_{A}, \text { and } \sigma^{k}(P)=a_{L} .
$$

If $P \in \mathcal{N}^{m, k}$ and $Q \in O P S^{m^{\prime}, k^{\prime}}$ (resp. $O P H^{m^{\prime}}$ ), then

$$
P Q \in O P S^{m+m^{\prime}, k+k^{\prime}} \quad\left(\text { resp. } O P H^{m+m^{\prime}, k+k^{\prime}}\right)
$$

and

$$
\sigma(P Q)-P_{\Sigma} \sigma(Q) \in S^{m+m^{\prime}, k+k^{\prime}+1}\left(\text { resp. } \mathscr{H}^{m+m^{\prime}-\frac{1}{2}(k+1)}\right)
$$


We shall also use the pseudo-differential operators with a parameter $\lambda \geq$ 1. By $S^{m}(\lambda)$, we denote the set of symbols such that for each $(\alpha, \beta)$, there is a positive constant $C_{\alpha \beta}$ such that

$$
\left|\partial_{x}^{\alpha} \partial \xi \beta a(x, \xi, \lambda)\right| \leq C_{\alpha \beta} \lambda^{m-|\beta|}, \forall(x, \xi) \in \mathbf{R}^{2 n}, \forall \lambda \geq 1 .
$$

By $\tilde{S}^{m}(\lambda)$, we denote the set of symbols such that for each $(\alpha, \beta)$, there is a positive constant $C_{\alpha \beta}$ such that

$$
\left|\partial_{x}^{\alpha} \partial \xi \beta a(x, \xi, \lambda)\right| \leq C_{\alpha \beta} \lambda^{m-\frac{1}{2}|\alpha+\beta|}, \forall(x, \xi) \in \mathbf{R}^{2 n}, \forall \lambda \geq 1 .
$$

By $S^{m, k}(\lambda)$, we mean the class of symbols characterized by the following: $a \in S^{m, k}(\lambda)$ if and only if $a$ is in $S^{m}(\lambda)$ outside of $\Sigma$ and every point of $\Sigma$ has a conic neighborhood in which for any $\alpha, \beta$ and any $|v| \geq 1$, there is a constant $\mathrm{C}_{\alpha, \beta}$ such that

$$
\left|\partial_{u}^{\alpha} \partial_{v}^{\beta} a\right| \leq C_{\alpha, \beta}|\lambda|^{m-|\beta|}\left(|u|+|\lambda|^{-1 / 2}\right)^{k-|\alpha|} .
$$

We set $\mathscr{H}^{m}(\lambda)=\bigcap_{j=0}^{\infty} S^{m-j, k-2 j}(\lambda)$. Then, we see that $a \in \mathscr{H}^{m}(\lambda)$ if and only if outside of $\Sigma, a \in S^{-\infty}(\lambda)$ and for any point $(x, \xi) \in \Sigma$, there is a conic neighborhood $\Theta$ such that $\forall \alpha, \beta, \gamma, \exists C_{\alpha \beta \gamma}$

$$
\left|u^{\alpha} \partial_{u}^{\beta} \partial_{v}^{r} a\right| \leq C_{\alpha \beta \gamma} \lambda^{m-|\gamma|+\frac{1}{2}|\beta|-\frac{1}{2}|\alpha|}
$$

for any $(v, u) \in \Theta$ and any $\lambda \geq 1$. We denote the classes of pseudo-differential operators corresponding to these classes by $\operatorname{OPS}^{m, k}(\lambda)$ and $O P H^{m}(\lambda)$.

Let

$$
N^{m, k}(\lambda)=S^{m}(\lambda) \cap S^{m, k}(\lambda) .
$$

By the similar argument to Proposition 3.2 and 3.5 in [B], we can prove the following two lemmata.

Lemma 2.1. If $P \in O P S^{m, k}(\lambda)$ and $Q \in O P S^{m^{\prime}, k^{\prime}}(\lambda)$, then

$$
P Q \in O P S^{m+m^{\prime}, k+k^{\prime}}(\lambda),
$$

and for any $N$,

$$
\sigma(P Q)-\sum_{|\alpha|<N} \frac{1}{\alpha !} \partial_{\xi}^{\alpha} \sigma(P) D_{x}^{\alpha} \sigma(Q) \in S^{m+m^{\prime}-N, k+k^{\prime}-2 N}(\lambda) .
$$

Lemma 2.2. If $P \in O P N^{m, k}(\lambda)$ and $Q \in O P S^{m^{\prime}, k^{\prime}}(\lambda)$, then

$$
\sigma(P Q)-\sum_{|\alpha| \leq k} \frac{1}{\alpha !} \partial_{\xi}^{\alpha} \sigma(P) D_{x}^{\alpha} \sigma(Q) \in S^{m+m^{\prime}-\frac{1}{2}, k+k^{\prime}}(\lambda)
$$


and

$$
\sigma(Q P)-\sum_{|\alpha| \leq k} \frac{1}{\alpha !} \partial_{\xi}^{\alpha} \sigma(Q) D_{x}^{\alpha} \sigma(P) \in S^{m+m^{\prime}-\frac{1}{2}, k+k^{\prime}}(\lambda) .
$$

By the symbol calculus in Lemma 2.1, we can easily show that the following result holds.

Lemma 2.3. Let $a \in S^{m, k}(\lambda)$ such that

$$
|a| \geq_{c} \lambda^{m} d_{\Sigma}^{k},(c>0)
$$

where $d_{\Sigma}(\lambda)=\left(|u|^{2}+\lambda^{-1}\right)^{\frac{1}{2}}$. Then, there is a symbol $b \in S^{-m,-k}(\lambda)$ such that

$$
\text { op }(a) \text { op }(b)-I d \in O P S^{-\frac{1}{2},-1}(\lambda)
$$

and

$$
\text { op }(b) \text { op }(a)-I d \in O P S^{-\frac{1}{2},-1}(\lambda) \text {. }
$$

Also, we shall use the following convention. For a family of operators $T=$ $\left(T_{1}, \cdots, T_{l}\right)$ and a finite sequence of positive integers $\alpha=\left(\alpha_{1}, \cdots, \alpha_{N}\right)$, where 1 $\leq \alpha_{j} \leq l$,

$$
T^{\alpha}=T_{\alpha_{1}} \cdots T_{\alpha_{N}} \text { and }|\alpha|=N .
$$

We denote the $L^{2}$-norm by $\|\cdot\|$ and its inner product by $(\cdot, \cdot)$ respectively. Finally, for two Banach spaces $E$ and $F$, we denote the space of bounded linear operators from $E$ to $F$ by $\mathscr{L}(E, F)$.

\section{Reduction to an $L^{2}$-inequality depending on a parameter}

We remark that if $P \in \mathcal{N}^{m, k}, P$ can be written as follows :

$$
P=\sum_{|\alpha| \leq k} A_{\alpha} U_{p}^{\alpha 1} \cdots U_{p}^{\alpha p}
$$

where $A_{\alpha} \in O P S_{1,0}^{m-\frac{1}{2}(k-|\alpha|)}(\Omega)$ and $U_{j}=u_{j}(x, D) \in \mathcal{N}^{0,1}$. Let $K$ be a compact subset of $\Omega$. Using a partition of unity such that

$$
\sum_{j} \phi_{j}(x, D)=I d \text { on } K
$$

we can reduce Theorem 1.1 to the following result.

Theorem 3.1. Let $\left(x_{0}, \xi_{0}\right)$ be a point of $\Sigma$. For a bounded neighborhood $V$ of $x_{0}$, there are an operator $\phi(x, D) \in O P S_{1,0}^{0}$ which is elliptic at $\left(x_{0}, \xi_{0}\right)$, posi. tive numbers $R$ and $C$ such that for any $|\operatorname{Im} \zeta|>R$ and $f \in C_{0}^{\infty}(V)$ 


$$
\begin{aligned}
& |\operatorname{Im} \zeta| \sum_{|\alpha|+j \leq m-1}\left\|\zeta^{j} \phi(x, D) U^{\alpha} f\right\|_{|\alpha|+\frac{1}{2}(m-1-j-|\alpha|)} \\
& \leq C\left\|P_{\zeta} f\right\|_{0}+C \sum_{|\alpha|+j \leq m-1}\left\|\zeta^{j} U^{\alpha} f\right\|_{|\alpha|+\frac{1}{2}(m-1-j-|\alpha|)},
\end{aligned}
$$

where

$$
P_{\zeta}=\zeta^{m}+\sum_{j=1}^{m} A_{j}(x, D) \zeta^{m-j}
$$

The proof shall be given in the following several sections. In this section, we shall reduce it to an $L^{2}$-estimate depending on a parameter $\lambda$. Let $\varphi \in C_{0}^{\infty}$ $\left(\mathbf{R}^{n}\right)$ be a non-negative function such that

$$
\operatorname{supp} \varphi \subset\left\{\xi \in \mathbf{R}^{n} ; 3 / 4 \leq|\xi| \leq 3\right\}
$$

and

$$
1=\chi(\xi)^{2}+\sum_{j \geq 0} \varphi\left(\xi / 2^{j}\right)^{2}
$$

where $\chi \in C_{0}^{\infty}\left(\mathbf{R}^{n}\right)$ is another function with its support contained in $\{|\xi| \leq 3 /$ $2\}$. Also, let $\tilde{\varphi}$ be another function having the same property as $\varphi$ such that $\tilde{\varphi}$ $=1$ on the support of $\varphi$. Let $\Theta$ be a conic neighborhood of $\left(x_{0}, \xi_{0}\right)$. We choose a $C^{\infty}$ and non-negative function $\psi(x, \xi)$ having its support in $\Theta$ such that $\psi$ $\left(x_{0}, \xi_{0}\right)=1$ and when $|\xi| \geq 1, \psi$ is homogeneous in $\xi$ of degree zero. Let $\tilde{\phi}$ be another function having the same property as $\phi$ such that $\tilde{\phi} \supset \supset \psi$, which means that $\tilde{\phi}=1$ on the support of $\phi$.

We can write

$$
P_{\zeta}=\zeta^{m}+\sum_{j=1|\alpha| \leq j}^{m} \sum^{m-j} A_{\alpha, j}(x, D) U^{\alpha},
$$

where the degree of $A_{\alpha, j} \leq \frac{1}{2}(j+|\alpha|)$. Let us denote the principal symbol of $A_{\alpha, j}$ by $a_{\alpha, j}$. For each $j=1, \cdots, p$, let $X_{j}$ be a pseudo-differential operator having its symbol $\sigma\left(X_{j}\right)$ such that

$$
\sigma\left(X_{j}\right)(x, \xi)=\tilde{\phi}(x, \xi)|\xi| u_{j}(x, \xi)
$$

and let $X_{j}(\lambda)$ be a pseudo-differential operator with a parameter $\lambda>1$ having its symbol $\sigma\left(X_{j}(\lambda)\right)(x, \xi)$ given by

$$
X_{j}(x, \xi, \lambda)=\tilde{\varphi}(\xi / \lambda) \sigma\left(X_{j}\right)(x, \xi) .
$$

Then, we can see that $X_{j}(\lambda) \in \operatorname{OPS}^{1}(\lambda)$.

We pick up the dominant part of $P_{\zeta}$ :

$$
P_{\zeta}(\lambda)=\zeta^{m}+\sum_{j=1}^{m} \sum_{|\alpha|=j} \zeta^{m-j} C_{\alpha, j} X(\lambda)^{\alpha}
$$

where $C_{\alpha, j}$ is a pseudo-differential operator with its symbol $c_{\alpha, j}$ 


$$
c_{\alpha, j}(x, \xi)=a_{\alpha, j}(x, \xi)|\xi|^{-j}
$$

if $|\xi| \geq 1$. Let $P_{\zeta}^{\prime}(\lambda)=\partial_{\zeta} P_{\zeta}(\lambda)$.

Theorem 3.2. For any $\varepsilon>0$, there are positive numbers $R_{0}$ and $C$ such that for any $|\operatorname{Im} \zeta|>R_{0}, f \in \mathscr{S}$ and $\lambda \geq 1$,

$$
\begin{aligned}
& |\operatorname{Im} \zeta| \sum_{|\alpha|+j=m-1}\left\|\zeta^{j} X(\lambda)^{\alpha} f\right\| \\
& \leq C\left\|P_{\zeta}(\lambda) f\right\|_{0}+\sum_{|\alpha|+j \leq m-2}(\varepsilon|\operatorname{Im} \zeta|+C)\left\|\zeta^{j} \lambda^{\frac{1}{2}(m-1-j-|\alpha|)} X(\lambda)^{\alpha} f\right\| .
\end{aligned}
$$

Granting this result, we can prove Theorem 3.1. The rest of this section is devoted to its proof.

Let

$$
\varphi_{\nu}(D)=\varphi\left(\frac{D_{x}}{2^{\nu}}\right), \lambda_{\nu}=2^{\nu} \text { and } f_{\nu}=\phi(x, D) \varphi_{\nu}(D) f
$$

We apply Theorem 3.2 to $f_{\nu}$. To estimate the commutator terms, we use the following basic inequalities. (c.f. [HN]) For each $l \leq m$ and $\alpha,|\alpha| \leq l$,

and

$$
\begin{aligned}
& \sum_{\nu>0} \lambda_{\nu}^{l-|\alpha|}\left\|\left\{\varphi_{\nu}(D) \phi(x, D) X^{\alpha}-X\left(\lambda_{\nu}\right)^{\alpha} \phi(x, D) \varphi_{\nu}(D)\right\} f\right\|^{2} \\
& \leq C \sum_{|\beta| \leq|\alpha|-1}\left\|X^{\beta} f\right\|_{(l-1-|\beta|) / 2}^{2},
\end{aligned}
$$

$$
C^{-1}\|f\|_{s / 2}^{2} \leq \sum_{\nu \geq 0}^{s}\left\|\lambda_{\nu}^{s} \varphi_{\nu}(D) f\right\|^{2} \leq C\|f\|_{s / 2}^{2}
$$

for any $f \in \mathscr{S}$.

Then,

$$
\sum_{\nu \geq 0} \lambda_{\nu}^{l-|\alpha|}\left\|X\left(\lambda_{\nu}\right)^{\alpha} f_{\nu}\right\|^{2} \leq C\left\{\left\|\phi(x, D) X^{\alpha} f\right\|_{(l-|\alpha|) / 2}^{2}+\sum_{|\alpha| \leq l-1}\left\|X^{\alpha} f\right\|_{(l-1-|\alpha|) / 2}^{2}\right\} .
$$

On the other hand, since the system of vector fields $\left\{X_{j}\right\}_{j=1}^{p}$ and their commutators with length less than or equal to two span the tangent space at each point in $\Sigma$, we have

$$
\|\phi(x, D) f\|_{s+\frac{1}{2}}^{2} \leq C_{s}\left(\sum_{j=1}^{p}\left\|\phi(x, D) X_{j}\right\|_{s}^{2}+\|f\|_{s}^{2}\right)
$$

for any $f \in \mathcal{\&}$. (c.f. $[\mathrm{BCN}]$ )

$$
\begin{aligned}
& \sum_{|\alpha| \leq m-2}\left\|\phi(x, D) X^{\alpha} f\right\|_{(m-1-|\alpha|) / 2}^{2} \\
& \leq C \sum_{|\alpha|=m-1}\left\|\phi(x, D) X^{\alpha} f\right\|^{2}+C \sum_{|\alpha| \leq m-2}\left\|X^{\alpha} f\right\|_{(m-2-|\alpha|) / 2}^{2} .
\end{aligned}
$$

If $|\zeta| \geq|\operatorname{Im} \zeta| \geq R_{0}$ and we take $R_{0}$ as a large enough number, we get

$$
|\operatorname{Im} \zeta| \sum_{j+|\alpha| \leq m-1}\left\|\phi X^{\alpha} \zeta^{j} f\right\|_{\frac{1}{2}(m-1-|\alpha|-j)}
$$




$$
\leq C\|\psi P f\|+C \sum_{j+|\alpha| \leq m-1}\left\|\zeta^{j} X^{\alpha} f\right\|_{\frac{1}{2}(m-1-|\alpha|-j)} .
$$

This completes the proof of Theorem 3.1.

The proof of Theorem 3.2 consists of two parts. One of them is to handle the case that $|\operatorname{Im} \zeta| \geq R_{1} \lambda^{\frac{1}{2}}$ or $|\operatorname{Re} \zeta| \geq R_{2} \lambda^{\frac{1}{2}}$ holds. This is carried out in the next section in which the assumptions $(A-2)$ and (A-3) play an important role. The other is the consideration devoted to the remaining case, i.e. $|\zeta| \leq R_{3} \lambda^{\frac{1}{2}}$. This is given in $\S 5$ in which the assumptions $(A-1)$ and $(A-4)$ play an essential role.

\section{Estimates in the hyperbolic zone}

In this section, we shall give the proof of Theorem 3.2 when $|\zeta| \geq R_{1} \lambda^{\frac{1}{2}}$ holds. Let $\tilde{X}_{j}(\lambda)$ be an operator having its symbol

$$
\tilde{X}_{j}(x, \xi, \lambda)=X_{j}\left(x / \lambda^{\frac{1}{2}}, \lambda^{\frac{1}{2}} \xi, \lambda\right)
$$

and similarly, $\bar{C}_{\alpha, j}(\lambda)$ be an operator with symbol

$$
\begin{aligned}
& \tilde{C}_{\alpha, j}(x, \xi, \lambda)=c_{\alpha, j}\left(x / \lambda^{\frac{1}{2}}, \lambda^{\frac{1}{2}} \xi\right), \text { and put } \\
& \tilde{P}_{\zeta}(\lambda)=\left(\lambda^{\frac{1}{2}} \zeta\right)^{m}+\sum_{j=1}^{m} \sum_{|\alpha|=j} \tilde{C}_{\alpha, j}(\lambda)\left(\lambda^{\frac{1}{2}} \zeta\right)^{m-j} \tilde{X}(\lambda)^{\alpha} .
\end{aligned}
$$

The proof of Theorem 3.2 consists of the following two parts.

Proposition 4.1. Suppose that $(A-2)$ holds. Let $\tilde{\zeta}=\lambda^{\frac{1}{2}} \zeta$. For any $\varepsilon>0$, there are a positive number $R_{1}$ and a positive constant $C$ such that if $|\operatorname{Im} \zeta| \geq R_{1}$,

$$
\begin{aligned}
& |\operatorname{Im} \zeta| \lambda^{\frac{1}{2}} \sum_{|\alpha|+j=m-1}\left\|\zeta^{j} \tilde{X}(\lambda)^{\alpha} f\right\| \\
& \leq C\left\|\tilde{P}_{\zeta}(\lambda) f\right\|+\sum_{|\alpha|+j \leq m-2}(\varepsilon|\operatorname{Im} \zeta|+C) \lambda^{\frac{1}{2} \|}\left\|\lambda^{\frac{1}{2}(m-1-|\alpha|-j)} \tilde{\zeta} j \tilde{X}(\lambda)^{\alpha} f\right\|
\end{aligned}
$$

for any $f \in \&\left(\mathbf{R}^{n}\right)$.

Proposition 4.2. Suppose that $(A-2)$ and $(A-3)$ hold. For any $\varepsilon>0$, there are positive numbers $R_{2}$ and $R_{3}$ such that if $|\operatorname{Im} \zeta| \leq R_{1},|\operatorname{Re} \zeta| \geq R_{2}$ and $|\operatorname{Im} \zeta|$ $\lambda^{\frac{1}{2}} \geq R_{3}$,

$$
\begin{aligned}
& |\operatorname{Im} \zeta| \lambda^{\frac{1}{2}} \sum_{|\alpha|+j=m-1}\left\|\zeta^{j} \tilde{X}(\lambda)^{\alpha} f\right\| \\
& \leq C\left\|\tilde{P}_{\zeta}(\lambda) f\right\|+\sum_{|\alpha|+j \leq m-2}\left(\varepsilon|\operatorname{Im} \zeta| \lambda^{\frac{1}{2}}+C\right)\left\|\lambda^{\frac{1}{2}(m-1-|\alpha|-j)} \zeta^{j} \tilde{X}(\lambda)^{\alpha} f\right\|
\end{aligned}
$$

for any $f \in \mathscr{S}\left(\mathbf{R}^{n}\right)$.

Let us begin with the proof of Proposition 4.1. We are going to estimate 


$$
\left(\tilde{Q}_{\zeta}(\lambda) f, f\right)=2 i \operatorname{Im}\left(\tilde{P}_{\zeta}(\lambda) f, \tilde{P}_{\zeta}^{\prime}(\lambda) f\right) .
$$

Let us denote the principal symbol of $\tilde{P}_{\zeta}$ by $\tilde{p}_{\zeta}$ :

$$
\tilde{p}_{\zeta}(x, \xi, \lambda, \zeta)=\left(\lambda^{\frac{1}{2}} \zeta\right)^{m}+\sum_{j=1}^{m} \sum_{|\alpha|=j} \tilde{C}_{\alpha, j}(x, \xi, \lambda)\left(\lambda^{\frac{1}{2}} \zeta\right)^{m-j} \tilde{X}^{\alpha}(x, \xi, \lambda) .
$$

Let

and

$$
\tilde{Q}_{\zeta}(\lambda)=\tilde{P}_{\zeta}^{\prime}(\lambda) * \tilde{P}_{\zeta}(\lambda)-\tilde{P}_{\zeta}(\lambda) * \tilde{P}_{\zeta}^{\prime}(\lambda)
$$

$$
d_{\zeta}(x, \xi, \lambda)=\sum_{j=1}^{p}\left|\tilde{X}_{j}(x, \xi, \lambda)\right|+\lambda^{\frac{1}{2}}|\zeta| .
$$

\section{Lemma 4.3. Denoting}

$$
-\tilde{q}_{\zeta}(x, \xi, \lambda)=2 \operatorname{Im}\left\{\overline{\partial_{\zeta} \bar{p}_{\zeta}^{\prime}}(x, \xi, \lambda) \tilde{p}_{\zeta}(x, \xi, \lambda)\right\},
$$

then we can find positive constants $C_{j, j}=1,2$ such that

$$
-\operatorname{Im} \zeta \lambda^{\frac{1}{2}} d_{\zeta}(x, \xi, \lambda)^{2 m-2} \leq C_{1} \tilde{q}_{\zeta}(x, \xi, \lambda)+C_{2}|\zeta| \lambda^{\frac{1}{2}}
$$

for any $(x, \xi, \lambda, \zeta), \lambda>1$.

Proof.

$$
\frac{p_{\zeta}^{\prime}}{p_{\zeta}}=\sum_{j=1}^{m} \frac{1}{\zeta-\lambda_{j}}=\sum_{j=1}^{m} \frac{\bar{\zeta}-\lambda_{j}}{\left|\zeta-\lambda_{j}\right|^{2}}
$$

and

$$
q_{\zeta}=2 \operatorname{Im}\left(\frac{p_{\zeta}^{\prime}}{p_{\zeta}}\left|p_{\zeta}\right|^{2}\right) .
$$

The characteristic roots $\lambda_{j}$ are homogeneous of degree 1 in $\mathfrak{X}$, where

$$
\mathfrak{X}_{j}=\tilde{X}_{j}(x, \xi, \lambda)
$$

and if $j \neq k$, for some $\delta>0,\left|\lambda_{j}-\lambda_{k}\right| \geq \delta|\mathfrak{X}|$. Hence, we can find positive constants $C_{j}$ such that

$$
\begin{aligned}
& q_{\zeta}=-\operatorname{Im} \zeta \sum_{j=1}^{m} \prod_{k \neq j}\left|\zeta-\lambda_{k}\right|^{2} \\
& \geq C_{1}|\operatorname{Im} \zeta|\langle\zeta ; \mathfrak{X}\rangle^{2 m-2}-C_{2}|\operatorname{Im} \zeta| .
\end{aligned}
$$

where $\langle\zeta ; \mathfrak{X}\rangle=\left(1+|\zeta|^{2}+|\mathfrak{X}|^{2}\right)^{\frac{1}{2}}$.

Let

$$
\tilde{A}_{R}=\left\{(x, \xi, \lambda, \zeta) \in \mathbf{R}^{2 n} \times \mathbf{R}_{+} \times \mathbf{C} ; d_{\zeta}(x, \xi, \lambda) \geq R \lambda^{\frac{1}{2}}, \lambda \geq 1\right\}
$$


and

$$
\tilde{B}_{R}=\left\{(x, \xi, \lambda, \zeta) \in \mathbf{R}^{2 n} \times \mathbf{R}_{+} \times \mathbf{C} ; d_{\zeta}(x, \xi, \lambda) \leq R \lambda^{\frac{1}{2}}, \lambda \geq 1\right\} .
$$

Also, let

$$
A_{R}=\left\{(x, \xi, \lambda) \in \mathbf{R}^{2 n} \times \mathbf{R}_{+} ; d_{0}(x, \xi, \lambda) \geq R \lambda^{\frac{1}{2}}, \lambda \geq 1\right\}
$$

and

$$
B_{R}=\left\{(x, \xi, \lambda) \in \mathbf{R}^{2 n} \times \mathbf{R}_{+} ; d_{0}(x, \xi, \lambda) \leq R \lambda^{\frac{1}{2}}, \lambda \geq 1\right\} .
$$

For each non-negative integer $k$, let

$$
N_{k}(f)=\sum_{|\alpha+\beta| \leq k}\left\|y^{\alpha} D_{y}^{\beta} f\right\|^{2}
$$

and

$$
N_{k, x, \xi}(f)=\sum_{|\alpha+\beta| \leq k}\left\|(y-x)^{\alpha}\left(D_{y}-\xi\right)^{\beta} f\right\|^{2} .
$$

Then, we note that any $f \in \mathscr{S}\left(\mathbf{R}^{n}\right)$,

$$
N_{k, x, \xi}\left(U_{x, \xi} f\right)=N_{k}(f),
$$

where

$$
U_{x, \xi}=e^{i y \cdot \xi} f(y-x) .
$$

Proposition 4.4. For any $\varepsilon>0$, there are a positive real number $R_{\varepsilon}$ and a positive contant $C$ such that if $|\operatorname{Im} \zeta| \geq R_{\varepsilon}$,

$$
\begin{aligned}
& \left|\left(\left\{\tilde{Q}(\lambda)-\tilde{q_{\zeta}}(x, \xi, \lambda)\right\} f, f\right)\right| \\
& \leq \varepsilon \lambda^{\frac{1}{2}}|\operatorname{Im} \zeta|\left(d_{\zeta}(x, \xi, \lambda)^{2 m-2}\|f\|^{2}+\lambda^{m-1} N_{2 m-1, x, \xi}(f)^{\frac{1}{2}}\left\|_{f}\right\|\right) \\
& +\lambda^{\frac{1}{2}}\left(C d_{\zeta}(x, \xi, \lambda)^{2 m-2}\|f\|+C \lambda^{m-1} N_{2 m-1, x, \xi}(f)^{\frac{1}{2}}\right) N_{4 m-2, x, \xi}(f)^{\frac{1}{2}}
\end{aligned}
$$

for any $f \in \&$.

Proof. Taylor's theorem says that for $f \in C^{k+1}([0,1])$,

$$
f(1)=\sum_{0}^{k} \frac{f^{(j)}(0)}{j !}+\frac{1}{k !} \int_{0}^{1} f^{(k+1)}(t)(1-t)^{k} d t .
$$

Applying this formula to the function

$$
f(t)=a(x+t(y-x), \xi+t(\eta-\xi)),
$$

we have

$$
a(y, \eta)=\sum_{|\alpha+\beta| \leq k} \frac{1}{\alpha ! \beta !} \partial_{x}^{\alpha} \partial \xi a(x, \xi)(y-x)^{\alpha}(\eta-\xi)^{\beta}
$$




$$
\begin{aligned}
& +(k+1) \sum_{|\alpha+\beta|=k+1} \frac{1}{\alpha ! \beta !}(y-x)^{\alpha}(\eta-\xi)^{\beta} \\
& \times \int_{0}^{1} \partial_{x}^{\alpha} \partial \xi \beta a(x+t(y-x), \xi+t(\eta-\xi))(1-t)^{k} d t .
\end{aligned}
$$

Let

$$
\begin{aligned}
& \mathfrak{X}_{j}^{(0)}(x, \xi, \lambda)=\tilde{X}(x, \xi, \lambda) \\
& \mathfrak{X}_{j}^{(1)}=\sum_{k=1}^{n}\left(\frac{\partial \tilde{X}_{j}}{\partial \xi_{k}}(x, \xi, \lambda)\left(D_{y_{k}}-\xi_{k}\right)+\frac{\partial \tilde{X}_{j}}{\partial x_{k}}(x, \xi, \lambda)\left(y_{k}-x_{k}\right)\right)
\end{aligned}
$$

and

$$
\tilde{X}_{j}(\lambda)=\mathfrak{X}_{j}^{(0)}(x, \xi, \lambda)+\mathfrak{X}_{j}^{(1)}(x, \xi, \lambda)+\mathfrak{X}_{j}^{(2)}(x, \xi, \lambda) .
$$

Also,

$$
\tilde{C}_{\alpha, j}=\mathfrak{X}_{0, \alpha, j}^{(0)}(x, \xi, \lambda)+\mathfrak{X}_{0, \alpha, j}^{(2)}(x, \xi, \lambda),
$$

where

$$
\mathfrak{X}_{0, \alpha, j}^{(0)}(x, \xi, \lambda)=\tilde{C}_{\alpha, j}(x, \xi, \lambda) .
$$

Then, Taylor's theorem and the estimate of the remainder terms imply that

$$
\mathfrak{X}_{j}^{(0)} \in \tilde{S}^{1}(\lambda)
$$

and the coefficients of the operator $\mathfrak{X}_{j}^{(k)}(x, \xi, \lambda)$ are in $\tilde{S}^{\frac{1}{2}(2-k)}(\lambda)$ if $k=1,2$.

Since

$$
\tilde{X}(\lambda) \in \tilde{S}^{1}(\lambda),
$$

the $L^{2}$-continuity theorem by Calderón-Vaillancourt enables us to write

$$
\begin{aligned}
& \left\|\left(\tilde{X}_{j}(\lambda)-\mathfrak{X}_{j}^{(0)}(x, \xi, \lambda)\right) f\right\| \leq C \lambda^{\frac{1}{2}} \sum_{|\alpha+\beta| \leq 1}\left\|(y-x)^{\alpha}\left(D_{y}-\xi\right)^{\beta} f\right\|, \\
& \left.\| \mathfrak{X}_{j}^{(2)}(x, \xi, \lambda)\right) f\left\|\leq C \sum_{|\alpha+\beta| \leq 2}\right\|(y-x)^{\alpha}\left(D_{y}-\xi\right)^{\beta} f \|,
\end{aligned}
$$

and

$$
\left\|\left(\tilde{C}_{\alpha, j}(\lambda)-\mathfrak{X}_{0, \alpha, j}(x, \xi, \lambda)\right) f\right\| \leq C \lambda^{-\frac{1}{2}} \sum_{|\alpha+\beta| \leq 1}\left\|(y-x)^{\alpha}\left(D_{y}-\xi\right)^{\beta} f\right\| .
$$

The operator $\tilde{Q}(\lambda)$ is a sum of terms such that

$$
\begin{aligned}
& \tilde{\zeta}^{k} \tilde{\zeta}^{j}\left(\tilde{X}(\lambda)^{\alpha}\right)^{*} \tilde{X}_{0, \alpha, j}(\lambda)^{*} \tilde{X}_{0, \beta, k}(\lambda) \tilde{X}(\lambda)^{\beta}-\tilde{\zeta}^{j} \tilde{\zeta}^{k}\left(\tilde{X}(\lambda)^{\beta}\right){ }^{*} \tilde{X}_{0, \beta, k}(\lambda)^{*} \tilde{X}_{0, \alpha, j} \\
& (\lambda) \tilde{X}(\lambda)^{\alpha} .
\end{aligned}
$$

It is seen that for $|\alpha|=l$,

$$
\prod_{k=1}^{l} \tilde{X}_{i_{k}}(\lambda)=\prod_{k=1}^{l} \mathfrak{X}_{i_{k}}^{(0)}
$$




$$
\begin{aligned}
& +\sum_{k=1}^{l}\left(\prod_{j \neq k} \mathfrak{X}_{i j}^{(0)}\right) \mathfrak{X}_{i k}^{(1)} \\
& +\sum_{k, i=1}^{l}\left(\prod_{j \neq i, k} \mathfrak{X}_{i j}^{(0)}\right) \mathfrak{X}_{i=}^{(1)} \mathfrak{X}_{i k}^{(1)}+\Re,
\end{aligned}
$$

where $\Re$ represents the sum of the remainder terms. After expanding each terms of $\tilde{Q}(\lambda)-\tilde{q_{\zeta}}(x, \xi, \lambda)$ as above, we can classify them into the following three groups:

1. the sum of terms having a factor $\operatorname{Im} \tilde{\zeta}$ but having no factor $\mathfrak{X}^{(2)}$,

2 . the sum of terms having no factor $\operatorname{Im} \tilde{\zeta}$ and having no factor $\mathfrak{X}^{(2)}$,

3 . the sum of remaining terms.

Using the fact that

$$
\tilde{X}^{*}(\lambda)-\tilde{X}(\lambda) \in O P \tilde{S}^{0}(\lambda)
$$

and

$$
\left[\mathfrak{X}_{j}^{(1)}, \mathfrak{X}_{k}^{(1)}\right] \in O P \tilde{S}^{1}(\lambda),
$$

we can estimate each group and see that for some positive constant $C$,

$$
\begin{aligned}
& \left|\left(\left\{\tilde{Q}(\lambda)-\tilde{q}_{\zeta}(x, \xi, \lambda)\right\} f, f\right)\right| \\
& \leq C|\operatorname{Im} \zeta| \lambda^{\frac{1}{2}} \sum_{j=0}^{2 m-3} \sum_{|\gamma| \leq 2 m-3-j} \sum_{|\alpha+\beta| \leq 2 m-2-j-|r|}|\tilde{\zeta}|^{j}\left|\tilde{X}^{\gamma}(x, \xi, \lambda)\right| \\
& \times \lambda^{\frac{1}{2}|\alpha+\beta|}\left|\left((y-x)^{\alpha}\left(D_{y}-\xi\right)^{\beta} f, f\right)\right| \\
& +C \sum_{j=0}^{2 m-3} \sum_{|r| \leq 2 m-3-j} \sum_{|\alpha+\beta| \leq 2 m-3-j-|r|}|\tilde{\zeta}|^{j}\left|\tilde{X}^{r}(x, \xi, \lambda)\right| \\
& \times \lambda^{\frac{1}{2}|\alpha+\beta|+1}\left|\left((y-x)^{\alpha}\left(D_{y}-\xi\right)^{\beta} f, f\right)\right| \\
& +C \sum_{j=0}^{2 m-3} \sum_{|r| \leq 2 m-2-j} \sum_{|\alpha+\beta| \leq 2 m-2-j-|r|}|\tilde{\zeta}|^{j}\left|\tilde{X}^{r}(x, \xi, \lambda)\right| \lambda^{\frac{1}{2}|\alpha+\beta|} \\
& \times \sum_{\left|\alpha^{\prime}+\beta^{\prime}\right| \leq 2(2 m-2-j-|r|-|\alpha+\beta|)+2}\left|\left((y-x)^{\alpha+\alpha^{\prime}}\left(D_{y}-\xi\right)^{\beta+\beta^{\prime}} f, f\right)\right|
\end{aligned}
$$

for any $(x, \xi) \in \mathbf{R}^{2 n}$, any $\lambda \geq 1$ and any $f \in \mathscr{S}\left(\mathbf{R}^{n}\right)$. Here, we have remarked the fact that for $j+|\gamma|=2 \mathrm{~m}-2$, the terms in the second group

$$
(\operatorname{Re} \tilde{\zeta})^{j}\left(\tilde{X}^{r}(x, \xi, \lambda) \mathfrak{X}_{j}^{(1)} f, f\right)
$$

were cancelled out.

The following is a well-known result. (c.f. $[\mathrm{HN}]_{2}$ )

Lemma 4.5. For each $l \in \mathbf{N}$ and any $\varepsilon>0$, there is a positive constant $C_{\varepsilon}$ such that we have

$$
\sum_{j=1}^{l} \delta^{j} N_{j}(f) \leq \varepsilon \delta^{l} N_{l+1}(f)+C_{\varepsilon} \delta^{1 / l} N_{0}(f)
$$

for any $\delta \in(0,1)$ and any $f \in \&\left(\mathbf{R}^{n}\right)$.

Proof. For any $\varepsilon>0$ there is a positive constant $C_{\varepsilon}$ such that for any $1 \leq j \leq l$, any $\boldsymbol{\Xi} \in \mathbf{R}_{+}$and any $\delta \in(0,1)$, 


$$
\delta^{j} \Xi^{j} \leq \varepsilon \delta^{l} \Xi^{l+1}+C_{\varepsilon} \delta^{1 / l}
$$

Let

$$
H=\left(-\Delta_{y}+|y|^{2}\right)^{\frac{1}{2}} .
$$

Then, for some positive constant $C$ such that

$$
C^{-1} N_{j}(f) \leq\left\|H^{j} f\right\|^{2} \leq C N_{j}(f) .
$$

Using the spectral resolution of the self-adjoint operator $H$ on $L^{2}\left(\mathbf{R}^{n}\right)$

$$
(H f, f)=\int_{0}^{\infty} t d\left(E_{t} f, f\right)
$$

we have

$$
(F(H) f, f) \leq(G(H) f, f)
$$

if $F(t) \leq G(t)$ for any $t \in \mathbf{R}_{+}$. Therefore,

$$
\left(a(H) *_{a}(H) f, f\right) \leq\left(b(H) *_{b}(H) f, f\right),
$$

if $a(t) \leq b(t)$ on $\mathbf{R}_{+}$. This completes the proof.

It then follows from (4.3) that

$$
\begin{aligned}
& \left|\left(\left\{\tilde{Q}(\lambda)-\tilde{q_{\zeta}}(x, \xi, \lambda)\right\} f, f\right)\right| \\
& \leq|\operatorname{Im} \zeta| \lambda^{\frac{1}{2}} \sum_{k=0}^{2 m-3} d_{\zeta}(x, \xi, \lambda){ }^{k} \lambda^{(2 m-2-k) / 2} N_{2 m-2-k, x, \xi}^{1 / 2}(f)\left\|_{f}\right\| \\
& +C \lambda^{\frac{1}{2}} \sum_{k=0}^{2 m-2} d_{\zeta}(x, \xi, \lambda){ }^{k} \lambda^{(2 m-2-k) / 2} N_{2 m-2-k, x, \xi}^{1 / 2}(f) N_{4 m-2, x, \xi}^{1 / 2}(f) .
\end{aligned}
$$

Taking $\delta=\left(\lambda^{\frac{1}{2}} / d_{\zeta}(x, \xi, \lambda)\right)^{2}$ in Lemma 4.5 , we have

$$
\begin{aligned}
& \sum_{k=0}^{2 m-3} d_{\zeta}(x, \xi, \lambda){ }^{k} \lambda^{\frac{1}{2}(2 m-2-k)} N_{2 m-2-k, x, \xi}(f) \\
& \leq \varepsilon \lambda^{\frac{1}{2}(2 m-2)} N_{2 m-1, x, \xi}(f)+\varepsilon d_{\zeta}(x, \xi, \lambda)^{2 m-2} N_{0}(f),
\end{aligned}
$$

for $(x, \xi, \lambda, \zeta) \in \tilde{A}_{R(\varepsilon)}$ if $R(\varepsilon)$ is chosen large enough such that

$$
C_{\varepsilon} \delta^{1 / l} \leq \varepsilon .
$$

This completes the proof of Proposition 4.4.

Lemma 4.6. Let $l \in \mathbf{N}$ For any $\varepsilon>0$, there are a positive real number $R$ and a positive constant $C$ such that if $(x, \xi, \lambda, \zeta) \in \tilde{A}_{R}$,

$$
\begin{gathered}
d_{\zeta}(x, \xi, \lambda)^{2 l}\|f\|^{2} \leq C \sum_{j+|\alpha|=l}\left\|\tilde{\zeta} \tilde{\zeta}^{j} \tilde{X}(\lambda)^{\alpha} f\right\|^{2}+\varepsilon \lambda^{l} N_{l+1, x, \xi}(f) \\
\sum_{j+|\alpha|=l}\left\|\tilde{\zeta} \tilde{\zeta}^{j}(\lambda)^{\alpha} f\right\|^{2} \leq C d_{\zeta}(x, \xi, \lambda)^{2 l}\|f\|^{2}+\varepsilon \lambda^{l} N_{l+1, x, \xi}(f)
\end{gathered}
$$

for any $f \in \mathscr{\varnothing}$. 
Proof. As in Chap.X in $[\mathrm{HN}]_{2}$, for any $\varepsilon>0$, there is a positive number $R_{\varepsilon}$ such that if $(x, \xi, \lambda) \in A_{R \varepsilon}$,

$$
\begin{aligned}
& \left\|\left\{\sum_{|\alpha|=l} X(\lambda)^{\alpha}-d_{0}(x, \xi, \lambda)^{2 l}\right\}_{f}\right\|^{2} \\
& \leq \varepsilon \lambda^{l} N_{l+1, x, \xi}(f)+\varepsilon d_{0}(x, \xi, \lambda)^{2 l} N_{0, x, \xi}(f) .
\end{aligned}
$$

On the other hand, if $(x, \xi, \lambda) \in B_{R}$,

$$
\left\|\left(\tilde{X}_{j}(\lambda)-\mathfrak{X}_{j}^{(0)}\right) f\right\|^{2} \leq \lambda N_{1, x, \xi}(f) .
$$

Hence, if $(x, \xi, \lambda) \in B_{R}$,

$$
\left\|\left\{\sum_{|\alpha|=l} X(\lambda)^{\alpha}-d_{0}(x, \xi, \lambda)^{2 l}\right\} f\right\|^{2} \leq C \lambda^{l} N_{l, x, \xi}(f) .
$$

Using Lemma 4.5 again, we see that for any $\varepsilon>0$, there is a positive number $R_{\varepsilon}$ such that

$$
\sum_{j+k \leq l-1}|\tilde{\zeta}|^{2 j} \lambda^{k} N_{k, x, \xi}(f) \leq \varepsilon|\tilde{\zeta}|^{2 l-2} N_{0}(f)+\varepsilon \lambda^{l-1} N_{l, x, \xi}(f)
$$

if $|\tilde{\zeta}| \geq R_{\varepsilon} \lambda \frac{1}{2}$. This completes the proof of Lemma 4.6 .

From Lemma 4.4, Proposition 4.3 and Lemma 4.6, it follows that

$$
\begin{aligned}
& |\operatorname{Im} \zeta| \lambda^{\frac{1}{2}} \sum_{j+|\alpha|=m-1}\left\|\tilde{\zeta}^{j} \tilde{X}(\lambda)^{\alpha} f\right\|^{2} \\
& \leq C|(\tilde{Q}(\lambda) f, f)|+\varepsilon|\operatorname{Im} \zeta| \lambda^{\frac{1}{2}+m-1} N_{2 m-1, x, \xi}(f)+C \lambda^{\frac{1}{2}+m-1} N_{4 m-2, x, \xi}(f)
\end{aligned}
$$

for any $f \in \mathscr{S}\left(\mathbf{R}^{n}\right)$ if $|\operatorname{Im} \tilde{\zeta}| \geq R_{\varepsilon} \lambda^{\frac{1}{2}}$.

To complete the proof of Proposition 4.1, we use a partition of unity as follows:

Let $\phi \in C_{0}^{\infty}\left(\mathbf{R}^{n}\right)$ such that

$$
\sum_{j \in Z^{n}} \phi(x-j)^{2}=1 \text {. }
$$

For $\mu=(p, q) \in \mathbf{Z}^{2 n}$ and $\delta>0$, let

$$
\left(x_{\mu}, \xi_{\mu}\right)=(p / \delta, q / \delta)
$$

and

$$
\phi_{\mu}^{\delta}(y, \eta)=\phi(\delta y-p) \phi(\delta \eta-q)
$$

Denoting the corresponding pseudo-differential operator by $T_{\mu}^{\delta}$, we have the following fundamental facts. (c.f. Lemma 4.9 in Chap.X, [HN])

1. For $f \in \&\left(\mathbf{R}^{n}\right), \sum_{\mu}\left\|T_{\mu}^{\delta} f\right\|^{2}=\|f\|^{2}$.

2. For any $(\alpha, \beta)$, there is a positive constants $C_{\alpha, \beta}(\delta)$ such that

$$
\sum_{\mu}\left\|\left(y-x_{\mu}\right)^{\alpha}\left(D_{y}-\xi_{\mu}\right)^{\beta} T_{\mu}^{\delta} f\right\|^{2} \leq C_{\alpha, \beta}(\delta)\left\|f_{f}\right\|^{2}
$$


for any $f \in \mathcal{\&}$

3. If $\tilde{Y}_{j}(\lambda) \in O P \tilde{S}^{1}, j=1, \cdots, q$, for some constant $C$ we have

$$
\sum_{\mu}\left\|\left(\operatorname{ad} \tilde{Y}_{1}(\lambda)\right) \circ \cdots \circ\left(\operatorname{ad} \tilde{Y}_{q}(\lambda)\right) T_{\mu}^{\delta}\right\|^{2} \leq C \delta \lambda^{q}\left\|_{f}\right\|^{2}
$$

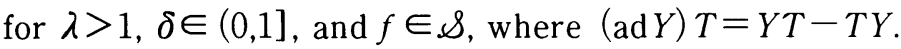

For $|\alpha|=m$ and two operators $Y, T$, there are positive constants $C_{\alpha \beta \gamma}$ such that

$$
\left[Y^{\alpha}, T\right]=\sum_{|\beta|+|\gamma|=m} C_{\alpha \beta \gamma}\left\{(\operatorname{ad} Y)^{\beta} T\right\} Y^{\gamma},
$$

so that

$$
\sum_{\mu}\left\|\left[\tilde{X}(\lambda)^{\alpha}, T_{\mu}^{\delta}\right] f\right\|^{2} \leq C \delta \sum_{|r| \leq m-1} \lambda^{m-|r|}\left\|\tilde{X}(\lambda)^{\gamma} f\right\|^{2}
$$

Summing up, taking $\delta$ small enough and letting $\varepsilon$ small enough such that

$$
C_{\alpha \beta}(\delta) \varepsilon<<1,
$$

we have

$$
\begin{aligned}
& |\operatorname{Im} \zeta| \lambda^{\frac{1}{2}} \sum_{j+|\alpha|=m-1}\left\|\tilde{\zeta}^{j} \tilde{X}(\lambda)^{\alpha} f\right\|^{2} \\
& \leq|(\tilde{Q}(\lambda) f, f)| \\
& \leq C\left\|\tilde{P}_{\zeta}(\lambda) f\right\|\left\|\tilde{P}_{\zeta}^{\prime}(\lambda) f\right\| \\
& +(\varepsilon|\operatorname{Im} \zeta|+C) \lambda^{\frac{1}{2}} \sum_{|\alpha|+j \leq m-1} \lambda^{\frac{1}{2}(m-1-|\alpha|-j)}\left\|\tilde{X}(\lambda)^{\alpha} \bar{\zeta}^{j} f\right\|^{2}
\end{aligned}
$$

Since

$$
\begin{aligned}
& \left\|\tilde{P}_{\zeta}(\lambda) f\right\|\left\|\tilde{P}_{\zeta}^{\prime}(\lambda) f\right\| \leq \frac{1}{2}\left\{\left(\varepsilon|\operatorname{Im} \zeta| \lambda^{\frac{1}{2}}\right)^{-1}\left\|\tilde{P}_{\zeta}(\lambda) f\right\|^{2}\right. \\
& \left.+\varepsilon|\operatorname{Im} \zeta| \lambda^{\frac{1}{2}} \sum_{|\alpha|+j \leq m-1}\left\|\tilde{X}(\lambda)^{\alpha} \zeta^{j} f\right\|^{2}\right\},
\end{aligned}
$$

we get

$$
\begin{aligned}
& |\operatorname{Im} \zeta| \lambda^{\frac{1}{2}} \sum_{|\alpha|+j=m-1}\left\|\tilde{\zeta}^{j} \tilde{X}(\lambda)^{\alpha} f\right\| \\
& \left.\leq C\left\|\tilde{P}_{\zeta}(\lambda) f\right\|+\sum_{|\alpha|+j \leq m-2}(C+\varepsilon|\operatorname{Im} \zeta|) \lambda^{\frac{1}{2}}\left\|\lambda^{\frac{1}{2}(m-1-|\alpha|-j)} \tilde{\zeta}^{j} \tilde{X}(\lambda)^{\alpha} f\right\|\right)
\end{aligned}
$$

for any $f \in \mathcal{S}\left(\mathbf{R}^{n}\right)$. Therefore, we get the desired inequality (4.1).

Now, we are going to prove Proposition 4.2. Let

$$
\mathfrak{X}_{j}^{(1)}(x, \xi, \lambda)\left(y, D_{y}\right)=S_{j}(x, \xi, \lambda)(y-x)+\mathrm{T}_{j}(x, \xi, \lambda)\left(D_{y}-\xi\right) .
$$

Then, $S$ and $T$ belong to the class $\tilde{S}^{\frac{1}{2}}(\lambda)$. By Taylor's theorem in the $(u, v)$ - coordinate, we can write

$$
S_{j}(x, \xi, \lambda)=S_{j}(0, v, \lambda)+\sum_{j=0}^{p} R_{j}(u, v, \lambda)
$$

and 


$$
T_{j}(x, \xi, \lambda)=T_{j}(0, v, \lambda)+\sum_{j=0}^{p} R_{j}^{\prime}(u, v, \lambda) .
$$

Here, $R_{j}$ and $R_{j}^{\prime}$ are in $\tilde{S}^{0}(\lambda)$. Since $u_{j} \sim \tilde{X}_{j}(\lambda)|\xi|^{-1} \lambda^{-\frac{1}{2}}$, the commutativity assumption in (A-3) gives that the second group in $\tilde{Q}(\lambda)-\tilde{q_{\zeta}}(x, \xi, \lambda)$ is estimated by

$$
\begin{aligned}
& \leq C d_{0}(x, \xi, \lambda) \sum_{j=0}^{2 m-3} \sum_{|r| \leq 2 m-3-j} \sum_{|\alpha+\beta|=2 m-2-j-|\gamma|}|\zeta|^{j}\left|\tilde{X}^{\gamma}(x, \xi, \lambda)\right| \\
& \times \lambda^{\frac{1}{2}|\alpha+\beta|}\left|\left((y-x)^{\alpha}\left(D_{y}-\xi\right)^{\beta} f, f\right)\right| .
\end{aligned}
$$

Therefore, if $|\operatorname{Im} \zeta| \leq R,|\zeta| \geq R_{2}$ and $|\zeta| \lambda^{\frac{1}{2}} \geq R_{3}$, then

$$
\begin{aligned}
& \left|\left(\left\{\tilde{Q}(\lambda)-\tilde{q}_{\zeta}(x, \xi, \lambda)\right\} f, f\right)\right| \\
& \leq\left\{\left(\varepsilon \lambda^{\frac{1}{2}}|\operatorname{Im} \zeta|+C\right) \lambda^{m-1} N_{2 m-1, x, \xi}(f)^{\frac{1}{2}}+\varepsilon d_{\zeta}(x, \xi, \lambda)^{2 m-2}\|f\|\right\} \\
& \times N_{4 m-2, x, \xi}(f)^{\frac{1}{2}} .
\end{aligned}
$$

Hence,

$$
\begin{aligned}
& |\operatorname{Im} \zeta| \lambda^{\frac{1}{2}} \sum_{l+|\alpha|=m-1}\left\|\tilde{\zeta}^{j} \tilde{X}(\lambda)^{\alpha} f\right\|^{2} \\
& \leq|(\tilde{Q}(\lambda) f, f)|+\left(\varepsilon|\operatorname{Im} \zeta| \lambda^{\frac{1}{2}}+C\right) \lambda^{m-1} N_{4 m-2, x, \xi}(f) .
\end{aligned}
$$

Let $\tilde{\phi} \in C_{0}^{\infty}\left(\mathbf{R}^{n}\right)$ be another function such that $\tilde{\phi}=1$ on the support of $\phi$. Let us define $\tilde{T}_{\mu}^{\delta}$ by similar way. Estimating carefully the commutator of $\tilde{Q}(\lambda)$ with $T_{\mu}^{\delta}$ by using the assumption (A-3) and Lemma 4.6 for $f=\tilde{T}_{\mu}^{\delta} f$, we have

$$
\begin{aligned}
& \sum_{\delta}\left(\left[\tilde{Q}(\lambda), T_{\mu}^{\delta}\right] \tilde{T}_{\psi}^{\delta} f, \tilde{T}_{\mu}^{\delta} f\right) \leq C(\delta)\left(\varepsilon \lambda^{1 / 2}|\operatorname{Im} \zeta|+C\right) \sum_{j+|\alpha| \leq m-1} \lambda^{m-1-|\alpha|-j} \\
& \quad \times\left\|\tilde{\zeta}^{j} \tilde{X}(\lambda)^{\alpha} f\right\|^{2},
\end{aligned}
$$

where the constant $C$ is independent of $\varepsilon$. Then, the same argument as in the Propositions 4.1 gives the desired result (4.2).

\section{Estimates in the elliptic zone}

In this section we shall prove the following result.

Theorem 5.1. Suppose that $(A-1)$ and $(A-4)$ hold. Let $\tilde{\zeta}=\lambda \frac{1}{2} \zeta$. For any positive number $R_{3}$, there is a positive number $R_{4}$ such that if $|\zeta| \leq R_{3}, \operatorname{Im} \zeta<0$ and $|\operatorname{Im} \zeta| \lambda^{\frac{1}{2}} \geq R_{4}$,

$$
\begin{aligned}
& |\operatorname{Im} \zeta| \lambda^{\frac{1}{2}} \sum_{|\alpha|+j=m-1}\left\|\tilde{\zeta}^{j} \tilde{X}(\lambda)^{\alpha} f\right\| \\
& \leq C\left(\left\|\tilde{P}_{\zeta}(\lambda) f\right\|+\sum_{|\alpha|+j \leq m-2}\left\|\lambda^{\frac{1}{2}(m-1-|\alpha|-j)} \tilde{\zeta}^{j} \tilde{X}(\lambda)^{\alpha} f\right\|\right)
\end{aligned}
$$


for any $f \in \mathscr{S}$.

We say that $a(\zeta, x, \xi) \in S_{\xi}^{m, k}(\lambda)$ if and only if $a$ is smooth in $(x, \xi)$ and holomorphic in $\zeta$ such that $a_{\zeta}=a(\zeta, x, \xi) \in S^{m, k}(\lambda)$ uniformly with respect to $\zeta \in B\left(\lambda \frac{1}{2} R_{3}\right)$, where $B(r)$ denotes the ball in $\mathbf{C}$ having the center 0 with radius $r$. Let us denote by $\mathscr{H}_{\zeta}^{m}(\lambda)$ the set of smooth functions $a(\zeta, v, u)$ which are holomorphic in $\zeta$ and smooth in $v$ and $u$ on

$$
B\left(R \lambda^{\frac{1}{2}}\right) \times \Sigma \times N
$$

such that for any $\alpha, \beta$ and $\gamma$, there is a positive constant $C_{\alpha \beta r}$ such that

$$
\left|u^{\alpha} \partial_{u}^{\beta} \partial_{v}^{r} a\right| \leq C_{\alpha \beta \gamma} \lambda^{m-|r|+\frac{1}{2}|\beta|-\frac{1}{2}|\alpha|}
$$

for any $(\zeta, v, u) \in B\left(R \lambda^{\frac{1}{2}}\right) \times \Sigma \times N$.

Let $\varphi$ and $\phi$ be two cutoff functions having the similar property to those of $\phi$ and $\phi$, respectively such that

$$
\varphi \subset \subset \tilde{\varphi} \text { and } \phi \subset \subset \tilde{\varphi} \text {. }
$$

Proposition 5.2. Suppose that (A-1) holds. Then, there is an operator $Q_{1, \zeta}(\lambda) \in O P S_{\zeta}^{-m,-m}(\lambda)$ such that

$$
\left\{Q_{1, \zeta}(\lambda) P(\lambda)-I d\right\} \psi(x, D) \varphi\left(D / \lambda^{\frac{1}{2}}\right)=R_{1, \zeta}(\lambda) \in O P \mathscr{H}_{\zeta}^{0}(\lambda) .
$$

Proof. We follow the similar argument to that in $[\mathrm{O}]_{2}$. In a neighborhood of $\Sigma$, the principal symbol $p_{\zeta}(x, \xi, \lambda)$ of $P_{\zeta}(\lambda)$ satisfies

$$
\left|p_{\zeta}(x, \xi, \lambda)\right| \geq C \lambda^{m} d_{\Sigma}(\lambda)^{m}
$$

if $\zeta \in B\left(\lambda \frac{1}{2} R_{3}\right)$ and $|\xi||u| \geq R \lambda^{\frac{1}{2}}$ for a large enough $R>0$. Set

$$
q_{3, \zeta}(x, \xi, \lambda)=\chi(x, \xi, \lambda) / p_{\zeta}(x, \xi, \lambda),
$$

where $\chi$ is a smooth function having its support contained in the set $\{|\xi||u| \geq$ $\left.R \lambda^{\frac{1}{2}}\right\}$ and taking value 1 if $|\xi \| u| \geq 2 R \lambda^{\frac{1}{2}}$. Then, by the symbol calculus, we see that

$$
\left\{\operatorname{op}\left(q_{3, \zeta}\right) P(\lambda)-I d\right\} \phi(x, D) \varphi(D / \lambda)=R_{3, \zeta}(\lambda) \in O P S_{\zeta}^{-\frac{1}{2},-1}(\lambda)
$$

and there exists a symbol $q_{4, \zeta} \in S_{\zeta}^{0,0}(\lambda)$ such that

$$
\text { op }\left(q_{4, \zeta}\right)-\sum_{j<l}\left(r_{3, \zeta}\right)^{j} \in O P S_{\zeta}^{-l / 2,-l}
$$

for any positive integer $l$. Let 


$$
Q_{1, \zeta}(\lambda)=\text { op }\left(q_{4, \zeta}\right) \text { op }\left(q_{3, \zeta}\right)
$$

Then,

$$
Q_{1, \zeta}(\lambda) P_{\zeta}(\lambda)-I d=R_{\zeta}(\lambda) \in \bigcap_{l} O P S_{\zeta}^{-l / 2, l}=O P H_{\zeta}^{0}(\lambda)
$$

Let

$$
\begin{aligned}
& \Re_{\zeta}^{m}=\left\{a(x, \xi, \lambda, \zeta) ; \forall \alpha, \beta, \gamma, \exists C,\left|u^{\alpha} \partial_{u}^{\beta} \partial_{v}^{\gamma} a\right| \leq C \lambda^{m}\left(|\operatorname{Im} \zeta| \lambda^{\frac{1}{2}}\right)^{-1-|r|}\right. \\
& \left.\times \lambda^{-\frac{1}{2}|\gamma|+\frac{1}{2}|\beta|-\frac{1}{2}|\alpha|}\right\} .
\end{aligned}
$$

Then, we have

Proposition 5.3. Suppose that $(A-1)$ and $(A-4)$ hold. Then, for any $R_{1, \zeta}(\lambda) \in O P \mathscr{H}_{\zeta}^{0}$, there is an operator $Q_{2, \zeta}(\lambda) \in O P \Re_{\zeta}^{-m}(\lambda)$ such that

$$
Q_{2, \zeta}(\lambda) P_{\zeta}(\lambda)-R_{1, \zeta}(\lambda) \in O P \Re_{\zeta}^{-1}(\lambda)
$$

Granting this result, we have

$$
\left\{\left(Q_{1, \zeta}(\lambda)+Q_{2, \zeta}(\lambda)\right) P_{\zeta}(\lambda)-I d\right\} \phi(x, D) \varphi(D / \lambda)=R_{2, \zeta}(\lambda) \in O P \Re_{\zeta}^{-1}(\lambda) .
$$

Therefore, the $L^{2}$-boundedness theorem due to Calderón-Vaillancourt, we can show that the inequality $(5.1)$ is valid if $R_{\mathbf{4}}$ is large enough. This finishes the proof of Theorem 5.1 .

The rest of this section is devoted to the proof of Proposition 5.3. First, we have

Lemma 5.4. $\quad$ For $Q(\lambda) \in O P \Re_{\zeta}^{-m}(\lambda)$,

$$
\left\{\sigma(Q(\lambda) P(\lambda))-P_{\Sigma}^{\prime} \sigma(Q(\lambda))\right\} \phi(\varepsilon / \lambda) \phi(x, \xi) \in \Re_{\zeta}^{-1}(\lambda),
$$

where

$$
P_{\Sigma}^{\prime}=\zeta^{m}+\sum_{j=0}^{m-1} \zeta^{j}\left(A_{m-j}\right)_{\Sigma}^{\prime}
$$

and $\left(A_{m-j}\right)_{\Sigma}^{\prime}$ are the operators having the form (2.3).

Proof. This can be shown by the similar argument to that in Proposition 5.5 in $[\mathrm{B}]$.

To complete the proof of Propsition 5.3, it suffices to show the following result.

Proposition 5.5. Suppose that $(A-1)$ and $(A-4)$ hold. Then, for any 
$r_{1, \zeta} \in \mathscr{H}_{\zeta}^{0}(\lambda)$, there is a symbol $q_{2, \zeta}(\lambda) \in \Re_{\zeta}^{-m}(\lambda)$ such that

$$
P_{\Sigma}^{\prime} q_{2, \zeta}(\lambda)=r_{1, \zeta}(\lambda) \text {. }
$$

When $|\zeta| \leq R_{3}$, we may consider the operator $\tilde{\mathscr{P}}_{x, \zeta}$ as a globally elliptic operator in a sense of Grushin. Let us recall some fundamental facts from [ $\mathrm{BGH}]$. Let $\omega$ be an open subset of $\mathbf{C}$ and $\Gamma=\omega \times \Sigma \ni \tau=(\zeta, v)$. Modifying the definition in $\S 2$, we say that $a(\zeta, v, u) \in C^{\infty}\left(\Gamma, S^{m}(N)\right)$ belongs to the class $S_{\zeta}^{m}(\Gamma \times N)$ if and only if $a$ is holomorphic in $\zeta$ and for any compact subset $K$ in $\Gamma$, any multi-index $\alpha, \beta$, there is a positive constant $C_{K, \alpha, \beta}$ such that

$$
\left|\partial_{v}^{\alpha} \partial_{u}^{\beta} a(\zeta, v, u)\right| \leq C_{K, \alpha, \beta}\langle u\rangle^{m-|\beta|}
$$

for $(\zeta, v) \in K$. Also, for $a \in S_{\zeta}^{m}(\Gamma, N)$ and $\tau=(\zeta, v) \in \Gamma$, set

$$
a_{\tau}(y)=a(\tau, y) \text { and } L_{\tau}(y)=L(v, y)
$$

and denote its corresponding operator by $a_{L}^{\tau}$. Then, we have the analogous result of Theorem 4.1 in [BGH].

Lemma 5.6. Let $a \in S_{\zeta, \text { reg }}^{m}(\Gamma \times N)$ is elliptic: i.e. for some positive number $\delta$,

$$
|a(\tau, u)| \geq \delta|u|^{m}
$$

for $\tau$ in a compact set if $|u|$ is large enough. If $a_{L}^{\tau}$ is invertible in $\&(E)$ for any $\tau \in$ $\Gamma$, then there is a symbol $b \in S_{\zeta, \text { reg }}^{-\mathrm{m}}(\Gamma \times N)$ such that

$$
a_{L} \cdot b_{L}=b_{L} \cdot a_{L}=I d .
$$

Proof. By $a \circ b=a_{A} b$, we can define a structure of algebra on $S^{\infty}(N)$. We denote it by $\mathscr{L}_{A}$. Since $a$ is elliptic, it has a parametrix $b^{\prime} \in S \bar{\zeta}^{m}(\Gamma \times N)$ and

$$
b^{\prime} \circ a-1 \in S \bar{\zeta}^{-\infty}(\Gamma \times N) .
$$

Hence, by the same argument in the proof of Theorem 4.1 in [BGH], we can reduce our assertion to the following result.

Lemma 5.7. Let $r \in S_{\zeta}^{-\infty}(\Gamma \times N)$. If $1+r_{r}$ is invertible for any $\tau \in \Gamma$, then the inverse has the form $\left(1+s_{\tau}\right)$ with $s \in S_{\zeta}^{-\infty}(\Gamma \times N)$.

Proof. Let $k$ be an integer such that $k>\frac{1}{2} \operatorname{dim} N$. For $M \in \mathbf{N}$ and a compact set $K$ of $\Gamma$, we define a family of semi-norms on $S_{\zeta}^{-\infty}(\Gamma \times N)$ by

$$
\|a\|_{M}^{K}=\sum_{2|\alpha|+j=m} \sup _{(\zeta, v) \in K}\left\|D_{v}^{\alpha} a\right\|_{E_{k+j}}
$$

where 


$$
\|f\|_{E_{s}}=\left\|\left(\left|D_{u}\right|^{2}+|u|^{2}\right)^{s / 2} f\right\|_{L^{2}(\mathbf{R})} .
$$

Then, by the same method as in the proof of Corollary 4.19 in [BGH], we can show that for a relatively compact open subset $U$ of $\Gamma$, there is a positive constant $C_{0}$ such that if

$$
\|a\|_{0}^{U}<1 / C_{0},
$$

then the sum $\sum_{j=1}^{\infty} a^{j}$ converges in $S \bar{\zeta}^{-\infty}(U \times N)$. Therefore, the same reasoning as the last part of $\S 4$ in [BGH], we can prove our assertion of Lemma 5.7.

From (2.4), it follows that for $(x, \xi) \in \Sigma$ and for

$$
\begin{aligned}
& a(\zeta, u, v)=\zeta^{m}+\sum_{j=0}^{m-1} T_{m-j}\left(A_{m-j}\right) \zeta^{j}, \\
& \mathscr{P}_{x, \zeta}(\zeta)=a_{L}, \text { and } P_{\Sigma}^{\prime}=a_{t_{A}},
\end{aligned}
$$

We note that $a_{L}$ is a quasi-homogeneous operator;

$$
\left(a_{L}\right)\left(\zeta \lambda^{\frac{1}{2}}, v \lambda, y / \lambda^{\frac{1}{2}}, \lambda^{\frac{1}{2}}, D_{y}\right)=\lambda^{\frac{1}{2} m}\left(a_{L}\right)\left(\zeta, v, y, D_{y}\right)
$$

and also

$$
\left(a_{t_{A}}\right)\left(\zeta \lambda^{\frac{1}{2}}, v \lambda, u / \lambda^{\frac{1}{2}}, \lambda^{\frac{1}{2}} D_{u}\right)=\lambda^{\frac{1}{2} m}\left(a_{t_{A}}\right)\left(\zeta, v, u, D_{u}\right) .
$$

The difficulty in the proof of Proposition 5.5 is to get a uniform estimate when $\zeta$ approaches to the real line. To do this, we introduce a special coordinate. Let $v$ be fixed. By a metaplectic transformation, we may assume that

$$
L(x, \xi)=\left(\tilde{y}_{1}, \cdots, \tilde{y}_{s}, \tilde{\eta}_{1}, \cdots, \tilde{\eta}_{s}\right)=(\tilde{y}, \tilde{\eta}),
$$

where $s=p / 2$. Then, we can view $a_{L}$ as a pseudo-differential operator depending parameter $\tau=(\zeta, v)$ :

$$
a_{L}=a_{\zeta, v}\left(\tilde{y}, D_{\tilde{y}}\right)=a_{\tau}\left(\tilde{y}, D_{\tilde{y}}\right)
$$

and the same is valid for $a_{t_{A}}$.

From the hypothesis $(\mathrm{A}-1)$, it follows that $a_{\tau}(\tilde{y}, \tilde{\eta})$ is an elliptic symbol and $a_{r}(\tilde{y}, \tilde{D})$ is a Fredholm operator with index 0 because the set of values of the principal symbol of $A_{m}(x, \xi)$ is contained in a half line of $\mathbf{R}$. In fact, the operator $a_{0, v}-\mu$ has a bounded inverse from $L^{2}\left(\mathbf{R}^{s}\right)$ to the space $H^{(m)}$ except for the discrete values of $\mu$ and the imbedding from $H^{(s)}$ to $H^{\left(s^{\prime}\right)}$ is compact if $s>s^{\prime}$, where

$$
H^{(m)}=\left\{f \in \mathscr{S}^{\prime}\left(\mathbf{R}^{s}\right) ; \forall|\alpha| \leq m,\left(D_{y}^{2}+|\tilde{y}|^{2}\right)^{m / 2} f \in L^{2}\left(\mathbf{R}^{s}\right)\right\} .
$$

By the ananytic Fredholm theorem (c.f. Theorem VI .14 in [RS]), we know that $\widetilde{a_{r}^{-1}}(\tilde{y}, \tilde{D})$ is a meromorphic function in $\zeta \in \mathbf{C}$ with range in $\mathscr{L}\left(L^{2}\left(\mathbf{R}^{s}\right)\right.$, $\left.H^{(m)}\right)$. 
Since

$$
a_{r}=\mathscr{P}_{x, \xi}
$$

if $(u, v)=(x, \xi)$ and $u=(y, \eta)$, and the hypothesis $(\mathrm{A}-4)$ is invariant under

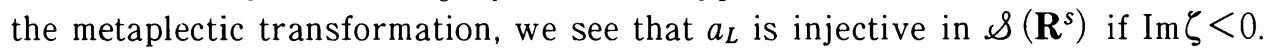
We note that $P_{\Sigma}$ is left-inversible and $P_{\Sigma}^{\prime}$ is right-inversible for such $\zeta$. Hence, $a_{r}^{-1}$ is also a meromorphic function in $\zeta \in \mathbf{C}$ with range in $\mathscr{L}\left(L^{2}\left(\mathbf{R}^{s}\right), L^{2}\left(\mathbf{R}^{s}\right)\right)$ and its poles are only in the set $\{\operatorname{Im} \zeta \geq 0\}$. We enumerate the poles $\left\{\zeta_{v}^{(j)}\right\}_{j=-\infty}^{\infty}$ lying on the real line:

$$
\zeta_{v}^{(j)}<\zeta_{v}^{(k)} \text { if } j<k
$$

Let $\mathscr{C}_{j}$ be a sufficiently small circle with center $\zeta_{v}^{(j)}$. Then, we see that for some positive integer $q$,

$$
\widetilde{a_{v}^{-1}}(\zeta)=\frac{1}{2 \pi i\left(\zeta-\zeta_{v}^{(j)}\right)^{q}} \oint_{\mathscr{C}_{1}}\left(\zeta-\zeta_{v}^{(j)}\right)^{q-1}{\tilde{a_{v}}}^{-1}\left(\zeta^{\prime}\right) d \zeta^{\prime}+\mathscr{O}\left(\left(\zeta-\zeta_{v}^{(j)}\right)^{-q+1}\right)
$$

if $\zeta$ is near $\zeta_{v}^{(j)}$. By the quasi-homogeneity, we can see that for each $j, \zeta_{v}^{(j)}$ is homogeneous of degree $1 / 2$ in $v$ and depends continuously on $v /|v|$ since in a locally, it is also a pole of a scalar-valued meromorphic function $\left(\tilde{a}_{\tau}^{-1} f, g\right)$ with some $f$ and $g \in \mathscr{S}\left(\mathbf{R}^{s}\right)$. By the second assumption of $(\mathrm{A}-4)$, we can conclude that the order of every poles is one. Locally, we can view a pole of a scalarvalued meromorphic function $F$ as a zero point of the holomorphic function 1/ $F$. Therefore, from the implicit function theorem, it follows that the poles $\zeta_{v}^{(j)}$ are smooth in $v /|v|$.

By Lemma 5.6, we can write

$$
a_{\tau}^{-1}=(b(\zeta, v, u))_{L},
$$

where $b(\zeta, v, u)$ is holomorphic in $\zeta$ if $\operatorname{Im} \zeta<0$ and has an analytic continuation near the points $\zeta_{v}^{(j)}$ which are isolated singular points of $b(\zeta, v, u)$. Using the inequality (1.2) again, we can conclude that the isolated singular points are poles with order 1 . We note that the quasi-homogeneity of $b$

$$
b\left(\zeta \lambda^{\frac{1}{2}}, v \lambda, \lambda^{-\frac{1}{2}} u\right)=\lambda^{-m / 2} b(\zeta, v, u)
$$

is important. Let $v_{0}$ be a fixed point such that $\left|v_{0}\right|=1$. The only $N$ poles $\zeta_{v_{0}}^{(j)}$ are in the domain $\left\{\zeta \in \mathbf{C} ;|\zeta| \leq R_{3}\right\}$. Let us consider the closed contours $\mathscr{C}_{j}, j=1$, $\cdots, N$

$$
\mathscr{C}_{j}=\left\{\left|\zeta-\zeta_{v_{0}}^{(j)}\right|=\delta\right\}
$$

where $\delta$ is a positive number. If $\delta>0$ is chosen as small enough, then the in- 
terior domains Int $\mathscr{C}_{j}$ are disjoint with each other and contain only one pole.

By the continuity of poles with respect to $v$, there is a small conic neighborhood $\mathcal{U}$ of $v_{0}$ such that if $v \in \mathcal{U}$, then

$$
\left|\zeta_{v /|v|}^{(j)}-\zeta_{v_{0}}^{(j)}\right| \leq \frac{1}{3} \delta
$$

From Lemma 5.6, it follows that for any $\alpha$ and $\beta$, there is a positive constant $C_{\alpha, \beta}$ such that if $v \in \mathcal{U}$ and

$$
\begin{aligned}
& \zeta \in \cap N_{j=1}^{N}\left\{\left|\zeta-\zeta_{v /|v|}^{(j)}\right| \geq \delta / 3\right\} \cap\left\{|\zeta| \leq R_{3}, \operatorname{Im} \zeta<0\right\} \\
& \text { or } \\
& \zeta \in \cup \cup_{j=1}^{N}\left\{2 \delta / 3 \geq\left|\zeta-\zeta_{v /|v|}^{(j)}\right| \geq \delta / 3\right\} \cap\left\{|\zeta| \leq R_{3}, \operatorname{Im} \zeta \geq 0\right\},
\end{aligned}
$$

then

$$
\left|\partial_{v}^{\alpha} \partial^{\beta}{ }_{u} b(\zeta, v, u)\right| \leq C_{\alpha, \beta}|v|^{-m}\left(|u|^{2}+|v|\right)^{-m / 2}
$$

for $|v| \geq 1$. We note that if $v \in \mathcal{U}$ and $\left|\zeta-\zeta_{v /|v|}^{(j)}\right| \leq \delta / 3$,

$$
\operatorname{dist}\left(\mathscr{C}_{j}, \zeta_{v /|v|}^{(j)}\right) \geq \frac{1}{3} \delta
$$

Hence, using the formula

$$
b(\zeta, v /|v|, u)=\frac{1}{2 \pi i\left(\zeta-\zeta_{v /|v|}^{(j)}\right)} \oint_{\mathscr{C}_{j}} b\left(\zeta^{\prime}, v /|v|, u\right) d \zeta^{\prime}+\Re_{j}^{\prime}(\zeta)
$$

where $\Re_{j}^{\prime}(\zeta)$ is a holomophic part near $\zeta_{v /|v|}^{(j)}$ and the quasi-homogeneous property, we see that $\forall \alpha$ and $\beta, \exists C_{\alpha, \beta}$ such that if $\zeta \in \cup_{j=1}^{N}\left\{\left|\zeta-\zeta_{v}^{(j)}\right| \leq \delta / 3\right\}, \operatorname{Im} \zeta$ $<0$,

$$
\left|\partial_{v}^{\alpha} \partial_{u}^{\beta} b(\zeta, v, u)\right| \leq C_{\alpha, \beta} \lambda^{-m}\left(|\operatorname{Im} \zeta| \lambda^{\frac{1}{2}}\right)^{-1-|\alpha|}|v|^{-\frac{1}{2}|\alpha|}\left(|u|^{2}+|v|\right)^{-m / 2-|\beta|}
$$

because

$$
\left|\partial_{v}^{\alpha} \zeta_{v}^{(j)}\right| \leq C_{\alpha}|v|^{\frac{1}{2}-|\alpha|} .
$$

By a finite covering of $\{\mathcal{U}\}$ on $B\left(\lambda^{\frac{1}{2}} R_{3}\right) \times \Sigma$, we can conclude that the estimate $(5.2)$ is valid for any $(\zeta, v, u) \in B\left(\lambda^{\frac{1}{2}} R_{3}\right) \times \Sigma \times E$.

Set $q_{2, \zeta}=b_{t_{A}} r_{1, \zeta}$. Then

$$
P_{\Sigma}^{\prime} q_{2, \zeta}=a_{t_{A}} b_{t_{A}} r_{1, \zeta}=r_{1, \zeta}
$$

and we can show that $q_{2,6} \in \Re^{m}(\lambda)$. This completes the proof of Proposition 5.5 .

\section{Examples}

We say that the Cauchy probelm for $P$ is well-posed at $\left(x_{0}, t_{0}\right) \in \Omega \times I$ if 
there is a neighborhood $\omega$ of $\left(x_{0}, t_{0}\right)$ such that for any $f \in C^{\infty}(\omega), g_{j} \in C^{\infty}(\omega \cap$ $\left.\left\{t=t_{0}\right\}\right), 0 \leq j \leq m-1$, the equation

$$
\left\{\begin{array}{l}
P u=f \quad \text { in } \omega \cap\left\{t>t_{0}\right\} \\
\partial_{t}^{j} u=g_{j} \text { on } \omega \cap\left\{t=t_{0}\right\}
\end{array}\right.
$$

has a unique solution $u(x, t) \in C^{\infty}\left(\omega \cap\left\{t \geq t_{0}\right\}\right)$. Then, it is easy to see that if $P$ and $P^{*}$ are maximally hyperbolic in $\Omega \times I$, then the Cauchy problem for $P$ is well-posed at each point of $\Omega \times I$. Conversely, if $P$ given by (1.1) is maximally hyperbolic, then the localized operator $\mathscr{P}_{x, \xi}(\zeta)$ is injective $\&\left(\mathbf{R}^{n}\right)$ if $\operatorname{Im} \zeta<0$ and $(x, \xi, t) \in \Sigma \times I$. This result shall be shown in the next section.

Is our injectivity condition satisfied by any operators? This is an interesting but a difficult problem. Few operators which satisfy it are known. In this section we consider this problem.

Let $t \in \mathbf{R}$ be a time variable and $x \in \mathbf{R}^{n+1}$ be a space variable. We consider a system $\left\{X_{1}, \cdots, X_{2 n}\right\}$ of $2 n$ vector fields on $\mathbf{R}^{n+1}$, given by

$$
X_{i}=D_{i}=(\sqrt{-1})^{-1} \partial / \partial x_{i}, 1 \leq i \leq n X_{n+i}=x_{i} D_{n+1}, 1 \leq i \leq n .
$$

The operators with which we are concerned have the following form

$$
\begin{aligned}
& P\left(D_{t}, X\right)=D_{t}^{m}+\sum_{j=0}^{m-1} a_{j}(X) D_{t}^{j}, \\
& a_{j}(X)=\sum_{|I|=m-j} a_{j, I} X^{I},
\end{aligned}
$$

where for $I=\left(i_{1}, \cdots, i_{p}\right) \in\{1, \cdots, 2 n\}^{p}$,

$$
X^{I}=X_{i_{1}} \cdots X_{i_{p}}, \quad a_{j, I} \in \mathbf{C}
$$

and

$$
\left\{a_{0}(\Xi)\right\}_{\Xi \in R^{2 n}} \subset \Gamma
$$

where $\Gamma$ is a half-line in the complex plane. For this operator, we can show the following negative result.

Theorem 6.1. Let $P$ be an operator as above. Suppose that $m$ is even and

$$
a_{0}(\Xi) \neq 0
$$

for any $\Xi \in \mathbf{R}^{2 n} \backslash 0$. Then, there exists a non-empty open set $U$ of $\mathbf{C}$ such that the Cauchy problem for the operator

$$
P+\alpha D_{n+1}^{m / 2}
$$

is not well-posed at the origin if $\alpha \in U$.

Proof. We suppose that for any $z \in \mathbf{C}$, the Cauchy problem for $Q(z)=P+$ $z D_{n+1}^{m / 2}$ is well-posed at the origin. Let 
where

$$
\mathscr{Q}_{\zeta,+}(z)=\zeta^{m}+\sum_{j+|\alpha|=m, j<m} a_{j, \alpha} \zeta^{j} \tilde{X}^{\alpha}+z
$$

$$
\tilde{X}=\left\{\begin{array}{l}
D_{i}, \text { if } 1 \leq i \leq n, \\
x_{i-n}, \text { if } n+1 \leq i \leq 2 n .
\end{array}\right.
$$

Then, it is easy to see that for any $z$ and $\zeta, \operatorname{Im} \zeta<0$,

$$
\operatorname{ker} \mathscr{Q}_{\zeta,+}(z)=\{0\} \text {. }
$$

Let $\zeta, \operatorname{Im} \zeta<0$ be fixed. From the argument as in the previous section, it follows that the inverse of $\mathscr{Q}_{\zeta,+}(z)$ is an entire function with range in $\mathscr{L}\left(L^{2}\right)$. Moreover, it is classical that the asymptotic behavior of the eigenvalues $\mu_{j}$ of the operator $\left(\mathscr{Q}_{0,+}\right) * \mathscr{Q}_{0,+}$ is as follows:

$$
\mu_{j} \sim c_{0} j^{m / n} \text { as } j \rightarrow \infty \text {. }
$$

(c.f.[S]) Therefore, we can conclude that the operator $\mathscr{Q}_{\zeta_{,}+}^{-1}$ belongs to so called $C_{p}$ class. (c.f.[DS]) This fact implies that for any $\varepsilon>0$ there is a constant $C$ such that,

$$
\left\|\mathscr{Q}_{\zeta,+}^{-1}(z)\right\| \leq C e^{|z|^{p+s}}
$$

for any $z \in \mathbf{C}$.

Since the principal symbol $\sigma_{0}\left(a_{0}\right)$ of $a_{0}(X)$ satisfies

$$
\left|\sigma_{0}\left(a_{0}\right)(x, \xi)\right| \geq \delta\langle x, \xi\rangle^{m}
$$

with $\delta>0, \mathscr{Q}_{\zeta,+}$ has a minimal growth on any ray contained in $\mathbf{C} \backslash \Gamma$ : i.e. there are a positive constant $C$ and a positive number $\rho_{0}$ such that

$$
\left\|\mathcal{Q}_{\zeta,+}(z) f\right\| \geq C\left\{\sum_{|\alpha| \leq m}\left\|\tilde{X}^{\alpha} f\right\|+\|z f\|\right\}
$$

if $z \in \Gamma$ and $|z| \geq \rho_{0}$. In fact, this is easily verified as in $\S 4$ in [O] ${ }_{2}$. Therefore we can apply the principle of Phragmén-Lindelöf to our operator and we see that $\left\|\mathcal{Q}_{\zeta_{,+}}^{-1}(z)\right\|$ is bounded on $\mathbf{C}_{z}$, which means that $\mathcal{Q}_{\zeta_{,+}}^{-1}(z)$ is independent of $z$. This is a contradition.

Let us define a subset $\mathcal{U}$ of $\mathbf{C}$ by

$$
\mathcal{U}=\left\{z \in \mathbf{C} ; \exists \zeta, \operatorname{Im} \zeta<0, \operatorname{ker} \mathscr{Q}_{\zeta,+}(z) \neq 0\right\} .
$$

Now, we are going to prove that $\mathcal{U}$ is open. Let $z_{0}$ be a point in $\mathcal{U}$. Then, there is a point $\zeta_{0}$ such that $\operatorname{Im} \zeta_{0}<0$ and

$$
\operatorname{ker} \mathscr{Q}_{\zeta_{0},+}\left(z_{0}\right) \neq 0 \text {. }
$$

Since $\zeta_{0}$ is a pole of the operator $\mathscr{Q}_{\zeta,+}^{-1}\left(z_{0}\right)$, for some positive integer $p$, we have

$$
\int_{\mathscr{Q}} \mathscr{Q}_{\zeta,+}^{-1}(z)\left(\zeta-\zeta_{0}\right)^{p} d \zeta \neq 0
$$


at $z=z_{0}$, where $\mathscr{C}=\left\{\left|\zeta-\zeta_{0}\right|=\varepsilon\right\}$ is a contour sufficiently near $\zeta_{0}$ and contained in $\{\operatorname{Im} \zeta<0\}$. On the other hand, for any $z, \mathscr{Q}_{\zeta,+}(z)^{-1}$ is also a meromorphic function in $\zeta$. Since for $\zeta \in \mathscr{C}, \mathscr{Q}_{\zeta,+}(z)$ is uniformly continuous in $z$ near $z_{0}$, we see that for some $p \geq 0$

$$
\int_{\mathscr{Q}^{2}} \mathscr{\zeta}_{,+}^{-1}(z)\left(\zeta-\zeta_{0}\right)^{p} d \zeta \neq 0
$$

if $\left|z-z_{0}\right|$ is sufficiently small. This means that the inner domain of $\mathscr{C}$ has a pole $\tilde{\zeta}$ of $\mathscr{Q}_{\zeta,+}^{-1}(z)$. Hence,

$$
\operatorname{ker} \mathscr{Q}_{\zeta,+}(z) \neq 0
$$

if $\left|z-z_{0}\right|$ is sufficiently small. This means that $z \in \mathcal{U}$.

Corollary 6.2. $\quad$ Suppose that the same condition as in Theorem 6.1 holds and for any $j, a_{j}(X)$ is formally self-adjoint. If the Cauchy problem for the operator

$$
P+\alpha D_{n+1}^{\mathrm{m} / 2}
$$

is well-posed, then $\alpha \in \mathbf{R}$.

Proof. Let

$$
\mathscr{Q}(\lambda)=\zeta^{m}+\sum_{j=0}^{m-1} a_{j}(\lambda) \zeta^{j}+\alpha \lambda^{m / 2},
$$

where

$$
a_{j}(\lambda)=\sum_{|I|=m-j} a_{j, I} X^{I}(\lambda)
$$

and

$$
\begin{array}{ll}
X_{i}=D_{i} & \text { if } 1 \leq i \leq n \\
X_{n+i}(\lambda)=x_{i} \lambda & \text { if } 1 \leq i \leq n .
\end{array}
$$

If $\zeta \in \mathbf{R}$, the formal self-adjointness of $a_{j}$ gives

$$
|(\mathscr{Q}(\lambda) f, f)| \geq\left|\operatorname{Im} \alpha\left\|\left.\lambda\right|^{k}\right\| f \|^{2}\right.
$$

for any $f \in \mathscr{S}(\mathbf{R})$. Therefore, if $\operatorname{Im} \alpha \neq 0$, then any real $\zeta$ is not eigenvalue of $\mathscr{Q}$ $( \pm 1)$ because the index of $\mathscr{Q}(\lambda)$ is zero. Since $\mathscr{Q}^{-1}$ is a meromorphic function on the whole space $\mathbf{C}_{\zeta}$, it has at least one pole which is a non-real point. This means that the injectivity breaks down at that point.

Let us denote by $H$ the following operator on $\mathbf{R}^{2}$

$$
D_{x}^{2}+x^{2} D_{y}^{2}
$$

For a positive integer $k$ and a complex number $c$, we consider the operator $P$ 
on $\mathbf{R}_{t} \times \mathbf{R}_{x} \times \mathbf{R}_{y}$

$$
P=\prod_{j=1}^{k}\left\{-D_{t}^{2}+a_{j} H\right\}-c D_{y}^{k},
$$

where $a_{j}$ are positive numbers such that

$$
a_{1}<a_{2}<\cdots<a_{k} .
$$

It is easily seen that this operator $P$ satisfies the conditions $(A-1)-(A-3)$.

Let $\Delta_{j}=\left[a_{j}, a_{j+1}\right]$ if $j>0, \Delta_{0}=\left[0, a_{1}\right]$ and

$$
f(\tau)=\prod_{j=1}^{k}\left(-\tau+a_{j}\right) .
$$

Then, we have

Proposition 6.3. When $k$ is odd, $P$ satisfies $(\mathrm{A}-4)$ if $c \in \mathbf{R}$ and

$$
\max _{0 \leq j \leq k-1, j=0 \bmod 2}\left\{\min _{\Delta j} f(t)\right\}<\varepsilon^{k} c<\min _{0 \leq j \leq k-1, j=1 \bmod 2}\left\{\max _{\Delta j} f(t)\right\},
$$

for any $\varepsilon= \pm 1$. When $k$ is even, $P$ satisfies $(A-4)$ if and only if $c \in \mathbf{R}$ and

$$
\max _{0 \leq j \leq k-1, j=1 \bmod 2}\left\{\min _{\Delta j} f(t)\right\}<c<\min _{0 \leq j \leq k-1, j=0 \bmod 2}\left\{\max _{\Delta j} f(t)\right\} .
$$

Proof. By Corollary 6.2, we may assume that $c \in \mathbf{R}$. Let

$$
\mathscr{P}_{\zeta}(\lambda)=\prod_{j=1}^{k}\left\{-\zeta^{2}+a_{j} \tilde{H}(\lambda)\right\}-c \lambda^{k},
$$

where

$$
\mathrm{H}(\lambda)=D_{x}^{2}+x^{2} \lambda^{2} .
$$

We take a family of Hermite functions $\left\{h_{k}(x)\right\}_{k=0}^{\infty}$ as an orthonormal basis of $L^{2}(\mathbf{R})$. Then,

$$
\tilde{\mathrm{H}}(1) h_{k}=(2 k+1) h_{k} \text {. }
$$

Especially,

$$
h_{0}=\pi^{-\frac{1}{2}} e^{-\frac{1}{2} x^{2}} .
$$

Since $h_{k} \in \mathscr{\&}(\mathbf{R})$ and for $f \in \mathscr{\&}(\mathbf{R})$,

$$
f=\sum_{k=0}^{\infty}\left(f, h_{k}\right) h_{k}
$$

$\mathscr{P}_{\zeta}(\lambda) f=0$ is equivalent to the system of equations

$$
\forall n, f_{n}\left(\zeta^{2}\right)-c \varepsilon^{k}=0,
$$

where $\varepsilon \lambda /|\lambda|= \pm 1$ and 


$$
f_{n}(t)=\prod_{j=1}^{k}\left(t-a_{j}(2 n+1)\right) .
$$

Let us denote the roots of the following equations

$$
f_{n}(\zeta)-c \varepsilon^{k}=0
$$

by $\lambda_{n, j}, 1 \leq_{j} \leq_{k}$. Then, we have

Lemma 6.4. If the condition in Proposition 6.3 holds, then for any $n, j$, $\lambda_{n, j}$ are positive and there is a positive constant $\delta$ such that for any $n$,

$$
\left|\lambda_{n, j}-\lambda_{n, j^{\prime}}\right|>\delta
$$

if $j \neq j^{\prime}$.

Proof. We prove the result when $k$ is odd. The other case can be proved in the same manner. Renaming the roots $\left\{a_{j}\right\}$ of $f(t)=0$ as follows:

$$
a_{1}<b_{1}<a_{2}<b_{2}<\cdots<a_{(k+1) / 2} \text {. }
$$

Then, we have

$$
\begin{aligned}
f(t) & \geq 0 \text { if } t \in\left[a_{j}, b_{j}\right] \\
& \leq 0 \text { if } t \in\left[b_{j}, a_{j+1}\right] .
\end{aligned}
$$

Let $a_{n}^{(j)}=(2 n+1) a_{j}$ and $b_{n}^{(j)}=(2 n+1) b_{j}$. For each $t \in I_{n}^{(j)}=\left[a_{n}^{(j)}, b_{n}^{(j)}\right]$, we assign a real number $\alpha \in[0,1]$ such that

$$
t=\alpha a_{n}^{(j)}+(1-\alpha) b_{n}^{(j)}=t_{\alpha, n}^{(j)}
$$

Since

$$
\begin{aligned}
& t_{\alpha, n}^{(j)}-a_{n}^{(j)}=(1-\alpha)\left(b_{j}-a_{j}\right)(2 n+1) \\
& b_{n}^{(j)}-t_{\alpha, n}^{(j)}=\alpha\left(b_{j}-a_{j}\right)(2 n+1)
\end{aligned}
$$

and

$$
b_{n+1}^{(j)}-a_{n+1}^{(j)}=(2 n+1)\left(b_{j}-a_{j}\right), a_{n+1}^{(j)}-b_{n+1}^{(j-1)}=(2 n+1)\left(a_{j}-b_{j-1}\right),
$$

we have

$$
f_{n}\left(t_{\alpha, n}^{(j)}\right) \geq f_{n-1}\left(t_{\alpha, n-1}^{(j)}\right)
$$

Hence,

$$
\max _{I_{n}^{\prime \prime}} f_{n} \geq \max _{I_{n}^{\prime \prime}-1} f_{n}
$$

Similar argument gives

$$
\max _{J_{N^{\prime \prime}}} f_{n} \leq \max _{J_{N^{\prime \prime}-1}} f_{n}
$$

where $J_{n}^{(j)}=\left[b_{n}^{(j-1)}, a_{n}^{(j)}\right]$ with $b_{n}^{(0)}=0$. Therefore, it suffices to show the result 
when $n=0$. Taking the shape of the graph $\left(t, f_{0}(t)\right)_{t \in \boldsymbol{R}}$ into consideration, we can get the conclusion.

Now, we can finish to prove Proposition 6.3. In fact, let $\zeta_{j}^{(n)}$ be the roots of

$$
f_{n}\left(\zeta^{2}\right)=c \varepsilon^{k} \text {. }
$$

Then, $\zeta_{j}^{(n)} \in \mathbf{R}$ and there is a positive constant $\delta$ such that for any $n$,

$$
\left|\zeta_{j}^{(n)}-\zeta_{l}^{(n)}\right| \geq 2 \delta>0
$$

if $j \neq l$. Therefore,

$$
\begin{aligned}
\left\|\mathscr{P}_{\zeta}(\varepsilon) g\right\|^{2} & =\sum_{n=1}^{\infty}\left|\left(f_{n}\left(\zeta^{2}\right)-c \varepsilon^{k}\right) c_{n}(g)\right|^{2} \\
& =\sum_{n=1}^{\infty} \prod_{j=1}^{2 k}\left|\operatorname{Re} \zeta-\zeta_{j}^{(n)}+i \operatorname{Im} \zeta\right|^{2}\left|c_{n}(g)\right|^{2} \\
& \geq|\operatorname{Im} \zeta|^{2} \delta^{2(2 k-1)}\|g\|^{2}
\end{aligned}
$$

for any $g \in \mathscr{\&}(\mathbf{R})$, where $c_{n}=\left(g, h_{n}\right)$.

Remark 6.5. $\quad$ From the result on the quasi-homogeneous operators in $[O]_{1}$, it follows that the well-posedness for the Cauchy proplem for $P$ in Proposition 6.3 gives the injectivity of $\mathscr{P}_{\zeta}( \pm 1)$ if $\operatorname{Im} \zeta<0$. Hence we can see that if the Cauchy problem for $P$ is well-posed, then $\varepsilon^{k} c$ must be in the closed interval with the same end points as those of the interval in Proposition 6.3.

Finally, we note that the condition $(\mathrm{A}-4)$ can be replaced by a simple but stronger condition that for any $(x, \xi) \in \Sigma$, the polynomial

$$
p_{f}(\zeta)=\left(\mathscr{P}_{x, \xi}(\zeta) f, f\right)
$$

is strictly hyperbolic uniformly in $f \in \mathscr{S}\left(\mathbf{R}^{n}\right) \backslash\{0\}$ : i.e. all $\operatorname{roots} \zeta_{j}(f)$ of $p_{f}(\zeta)$ $=0$ are real and there is a positive number $\delta$ such that for any $f \in \mathscr{\&}\left(\mathbf{R}^{n}\right) \backslash$ $\{0\}$,

$$
\left|\zeta_{j}(f)-\zeta_{k}(f)\right| \geq \delta>0
$$

if $j \neq k$. But we do not know what operators satisfy this condition except for $m$ $=2$.

\section{Normally hyperbolic operators}

In this section, we shall prove the injectivity condition is necessary for $P$ to be maximally hyperbolic. In fact, we can prove a stronger result. To do this, we define a weaker notion as follows. We say that $P$ is normally hyperbolic if and only if for any compact subset $K$ of $\Omega$, there are a real number $\gamma_{0}$ and a positive constant $C$ such that

$$
\gamma^{m}\left\|e^{-r t} u\right\| \leq C\left\|e^{-r t} P u\right\|,
$$


if $\gamma>\gamma_{0}$ and $u \in C_{0}^{\infty}(K \times I)$. We note that if $P$ is maximally hyperbolic, then $P$ is normally hyperbolic. In fact, it is well-known that there is a real number $\gamma_{0}$ such that

$$
\gamma\left\|e^{-r t} u\right\| \leq C\left\|e^{-\gamma t} D_{t} u\right\|
$$

for $u \in C_{0}^{\infty}(\Omega \times I)$ if $\gamma>\gamma_{0}$. Now, we can show the following result.

Theorem 7.1. Let $P$ be an operator given by (1.1). If $P$ is normally hyperbolic, then for any point $(x, \xi) \in \sum$, the operator $\mathscr{P}_{x, \xi}(\zeta)$ is injective in $\&$ $\left(\mathbf{R}^{n}\right)$ for any $\zeta, \operatorname{Im} \zeta<0$.

Proof. The proof is carried out by the localization method of [H]. Let $x_{0}=t$, $\xi_{0}=\tau$ and

$$
x=\left(x_{0}, \cdots, x_{n}\right)=\left(x_{0}, x^{\prime}\right), \xi=\left(\xi_{0}, \cdots, \xi_{n}\right)=\left(\xi_{0}, \xi^{\prime}\right) .
$$

Let $N=(1,0, \cdots, 0) \in \mathbf{R}^{n+1}$. For a compact subset $K$ of $\Omega$, let $\varphi \in C_{0}^{\infty}(\Omega)$ be equal to 1 in a neighborhood of $K$. For a fixed point $(x, \xi) \in \Sigma$, let

$$
\begin{aligned}
f(y) & =\varphi(y) \phi\left((y-x)|\xi| \frac{1}{2}\right) e^{i y \cdot \xi+r y_{0}} \\
& =\varphi(y) v(y) e^{r y_{0}} .
\end{aligned}
$$

Putting this function into the inequality (7.1), we have

$$
\gamma^{m}\|\varphi v\| \leq C\left\|P_{r}(y, D) \varphi v\right\|,
$$

where

$$
P_{\gamma}=P\left(y, D_{0}+i \gamma N, D^{\prime}\right) .
$$

We can write

$$
P_{\gamma} \varphi v=q(y, D+i \gamma N) v,
$$

where $q(y, \xi+i \gamma N) \sim p(y, \xi+i \gamma N)$ when $y \in K$ and

$$
\left\|q_{r}(y, D) v\right\|=\|q(y, D+\tilde{\xi}) w\|
$$

where $\tilde{\xi}=\left(\xi_{0}+i \gamma, \xi^{\prime}\right)$ and $w(y)=\phi\left((y-x)|\xi|^{\frac{1}{2}}\right)$. The Taylor expansion at $\tilde{\xi}$ up to order $m+1$ gives

$$
\begin{aligned}
& \left\|q_{r}(y, D) v\right\| \leq\left\|\sum_{|\alpha| \leq m+1} q^{(\alpha)}(y, \tilde{\xi}) D^{\alpha} w / \alpha !\right\| \\
& +C|\xi|^{-m+1} \sum_{|\alpha|=m+1}\left\|D^{\alpha} w\right\| .
\end{aligned}
$$

Using the Taylor expansion at $x$

$$
\begin{aligned}
& q^{(\alpha)}(y, \tilde{\xi})=\sum_{|\beta| \leq m} q_{(\beta)}^{(\alpha)}(x, \tilde{\xi})(y-x)^{\beta} D^{\alpha} w / \beta ! \\
& +(m+1) \sum_{|\beta|=m+1}(y-x)^{\beta} \int_{0}^{1} q_{(\beta)}^{(\alpha)}(x+t(y-x), \tilde{\xi})(1-t)^{m} d t / \beta !
\end{aligned}
$$


and the fact that

$$
\left\|(y-x)^{\beta} D^{\alpha} w\right\|=|\xi|^{-(n+1) / 4+\frac{1}{2}(|\alpha|-|\beta|)}\left\|y^{\beta} D^{\alpha} \psi\right\|,
$$

we have

$$
\begin{aligned}
& \gamma^{2 m}\|\varphi v\|^{2} \leq C\left\|q_{r} v\right\|^{2} \\
& \leq\left.\left. C \int\left|\sum_{|\alpha+\beta| \leq m} \frac{1}{\alpha ! \beta !} q_{(\beta)}^{(\alpha)}(x, \tilde{\xi})\right| \xi\right|^{\frac{1}{2}(|\alpha|-|\beta|)} y^{\beta} D^{\alpha} \psi(y)\right|^{2} d y|\xi|^{(n+1) / 2} \\
& +C(1+|\xi|)^{-1} N_{m+1}(\phi)|\xi|^{(n+1) / 2}
\end{aligned}
$$

for any $\phi \in C_{0}^{\infty}\left(\mathbf{R}^{n+1}\right)$. Let

$$
q=\sum_{j=0}^{m} \zeta^{j} q_{m-j}\left(x, \xi^{\prime}\right)
$$

and

$$
q_{k}\left(x, \xi^{\prime}\right) \sim \sum_{j=0}^{\infty} q_{k, j}\left(x, \xi^{\prime}\right)
$$

where $q_{k, j} \in S_{1,0}^{k-1}$. Then, from

$$
\left(p_{k, j}\right)\left(\begin{array}{l}
\alpha \\
\beta
\end{array}\right)\left(x, \xi^{\prime}\right) \neq 0 \text { if }|\alpha+\beta|+2 j=k
$$

it follows that

$$
\left(q_{k, j}\right)_{\left(\begin{array}{l}
\alpha \\
\beta
\end{array}\right)}^{(x,}\left(x, \xi^{\prime}\right)|\xi|^{\frac{1}{2}(|\alpha|-|\beta|)} \sim|\xi|^{\frac{1}{2} k} .
$$

We note that

$$
|\xi|^{\frac{1}{2}(n+1)}\|\varphi v\|^{2} \rightarrow\|\phi\|^{2}
$$

as $|\xi| \rightarrow \infty$. Let $\gamma=\tilde{\gamma}|\xi|^{\frac{1}{2}}, \operatorname{Im} \gamma_{0}<0$. Dividing (7.2) by $|\xi|^{\frac{1}{2}(m-n-1)}$ and letting $|\xi| \rightarrow \infty$, we have

$$
\tilde{\gamma}^{2 m}\|\tilde{\phi}\|^{2} \leq C\left\|\mathscr{P}_{x, \xi^{\prime}}\left(\eta_{0}+i \tilde{\gamma}\right) \tilde{\phi}\right\|^{2}
$$

for any $\phi \in C_{0}^{\infty}\left(\mathbf{R}^{n+1}\right)$ if $\left(x, \xi^{\prime}\right) \in \Sigma,|\xi|=1$. Here,

$$
\tilde{\phi}=\tilde{\phi}\left(\eta_{0}, x^{\prime}\right)
$$

denotes the Fourier transform of $\phi$ with respect to the variable $x_{0}$. For $\chi \in C_{0}^{\infty}$ (R) with $\|\hat{\chi}\|=1$, we set

$$
\hat{\chi}_{\varepsilon}\left(\eta_{0}\right)=\varepsilon^{\frac{1}{2}} \hat{\chi}\left(\varepsilon\left(\eta_{0}-\eta_{0}^{(0)}\right)\right) .
$$

For any $f \in \mathscr{S}\left(\mathbf{R}^{n}\right)$, let

$$
\tilde{\phi}_{\varepsilon}(x)=\hat{\chi}_{\varepsilon}\left(\eta_{0}\right) \phi_{q}\left(x^{\prime}\right) \text {, }
$$

where 


$$
\phi_{q}\left(x^{\prime}\right)=\tau\left(x^{\prime} / q\right) f\left(x^{\prime}\right)
$$

with $\tau \in C_{0}^{\infty}\left(\mathbf{R}^{n}\right)$ satisfying

$$
\tau\left(x^{\prime}\right)=1 \text { on }|x| \leq 1 \text {. }
$$

Using

$$
\left\|\left\{\mathscr{P}_{x, \xi^{\prime}}\left(\eta_{0}+i \tilde{\gamma}\right)-\mathscr{P}_{x, \xi^{\prime}}\left(\eta_{0}^{(0)}+i \tilde{\gamma}\right)\right\} \tilde{\phi}_{\varepsilon}\right\| \rightarrow 0 \text { as } \varepsilon \rightarrow 0 \quad \text { and }
$$

taking $\varepsilon \rightarrow+0$, we have

$$
\tilde{\gamma}^{2 m}\left\|\phi_{q}\right\|^{2} \leq C\left\|\mathscr{P}_{x, \xi^{\prime}}\left(\eta_{0}^{(0)}+i \tilde{\gamma}\right) \phi_{q}\right\|^{2} .
$$

Letting $q \rightarrow \infty$ for the obtained inequality, we can conclude that the injectivity in $\mathscr{S}$ of the operator $\mathscr{P}_{x, \xi^{\prime}}(\zeta)$ holds if $\operatorname{Im} \zeta<0$ and $\left(x, \xi^{\prime}\right) \in \Sigma$.

\section{Department of Mathemetics KYOTO UNIVERSITY}

\section{References}

[BCN] P. Bolley, J. Camus and J. Nourrigat, La condition de Hörmander-Kohn pour les opérateurs pseudo-différentiels, Comm. P. D. E., 8(1982), 197-221.

[B] L. Boutet de Monvel, Hypoelliptic operators with double characteristics and related pseudodifferential operators, Comm. Pure Apple. Math. 27 (1974), 585-639.

[BGH] L. Boutet de Monvel, A. Grigis and B. Helffer, Paramétrixes d'opérateurs pseudo-différentiells a caractéristiques multiples, Asterique, 34-35(1976), 93-121.

[DS] N. Dunford and J. T. Schwartz, Linear operators II. Interscience 1963.

$[\mathrm{HN}]_{1}$ B. Helffer and J. Nourrigat, Caracterisation des opérateurs hypoelliptiques homogènes invariants à gauche sur un groupe de Lie nilpotent gradué, Comm. in P. D. E. 4 (1976) 899958.

$[\mathrm{HN}]_{2}$ B. Helffer and J. Nourrigat, Hypoellipticité maximale pour des opérateurs polynomes de champs de vecteurs, Birkhäuser 1985.

[H] L. Hörmander, Psuedo-differential operators and non-elliptic boundary problems, Ann. of Math., 83(1966), 129-209.

$[0]_{1}$ T. Ōkaji, The Cauchy problem for non-effectively hyperbolic differential operators and their product, J. Math. Kyoto Univ., 29 (1989), 317-340.

[]$_{2}$ T. Ökaji, Equations of evolution on the Heisenberg group I, J. Math. Kyoto Univ., 32 (1992), 749-761.

$[0]_{3}$ T. Ōkaji, Equations of evolution on the Heisenberg group II , J. Math. Kyoto Univ., 33 (1993), 973-988.

[RS] M. Reed and B. Simon, Functional analysis, I, Revised and Enlarged Edition, Academic Press 1980.

[S] M. A. Shubin, Pseudo-differential operators and spectral theory (Translation in English), Springer-Verlag 1987. 